\title{
Properties of Mass-Loading Shocks 2. Magnetohydrodynamics
}

\author{
G. P. ZANK AND S. OUGHTON \\ Bartol Research Institute, University of Delaware, Newark \\ F. M. Neubauer \\ Institut für Geophysik und Meteorologie, Universität Köln, Cologne, Germany \\ G. M. WEBB
}

Department of Planetary Sciences, University of Arizona, Tucson

\begin{abstract}
The one-dimensional magnetohydrodynamics of shocked flows subjected to significant mass loading are considered. Recent observations at comets Giacobini-Zinner and Halley suggest that simple nonreacting MHD is an inappropriate description for active cometary bow shocks. The thickness of the observed cometary shock implies that mass loading represents an important dynamical process within the shock itself, thereby requiring that the Rankine-Hugoniot condition for the mass flux possess a source term. In a formal sense, this renders mass-loading shocks qualitatively similar to combustion shocks, except that mass loading induces the shocked flow to shear. Nevertheless, a large class of stable shocks exist, identified by means of the Lax conditions appropriate to MHD. Thus mass-loading shocks represent a new and interesting class of shocks, which, although found frequently in the solar system, both at the head of comets and, under suitable conditions, upsteam of weakly magnetized and nonmagnetized planets, has not been discussed in any detail. Owing to the shearing of the flow, mass-loading shocks can behave like switch-on shocks regardless of the magnitude of the plasma beta. Thus the behavior of the magnetic field in mass-loading shocks is significantly different from that occurring in nonreacting classical MHD shocks. It is demonstrated that there exist two types of mass-loading fronts for which no classical MHD analogue exists, these being the fast and slow compound mass-loading shocks. These shocks are characterized by an initial deceleration of the fluid flow to either the fast or the slow magnetosonic speed followed by an isentropic expansion to the final decelerated downstream state. Thus these transitions take the flow from a supersonic to a supersonic, although decelerated, downstream state, unlike shocks which occur in classical MHD or gasdynamics. It is possible that such structures have been observed during the Giotto-Halley encounter, and a brief discussion of the appropriate Halley parameters is therefore given, together with a short discussion of the determination of the shock normal from observations. A further interesting new form of mass-loading shock is the "slow-intermediate" shock, a stable shock which possesses many of the properties of intermediate MHD shocks yet which propagates like a slow mode MHD shock. An important property of mass-loading shocks is the large parameter regime (compared with classical MHD) which does not admit simple or stable transitions from a given upstream to a downstream state. This suggests that it is often necessary to construct compound structures consisting of shocks, slip waves, rarefactions, and fast and slow compound waves in order to connect given upstream and downstream states. Thus the Riemann problem is significantly different from that of classical MHD.
\end{abstract}

\section{INTRODUCTION}

Ever since Biermann [1951] inferred the existence of the solar wind from the properties of cometary tails, investigation of the solar wind-cometary atmosphere interaction has led to the discovery of many interesting and important phenomena in space plasma physics. Photoionization of the extensive neutral cometary coma leads to mass loading of the solar wind. Axford [1964] introduced an MHD description to model the cometary atmosphere-solar wind interaction, and, on this basis, Biermann et al. [1967] developed a relatively tractable, one-dimensional hydrodynamical model to describe supersonic mass-loaded plasma flow. The model, which demonstrates much of the basic physics of cometary atmospheres, has proved remarkably durable, and many of

Copyright 1992 by the American Geophysical Union.

Paper number 92JA00497. 0148-0227/92/92JA-00497\$05.00 the essential features of mass-loading flows are understood easily on this basis. It was recognized that mass loading would decelerate a supersonic flow and that this deceleration continued smoothly only until some time before a critical mass-loading rate was achieved. Biermann et al. [1967] (BBS) showed that a shock must form in the accreting supersonic flow before the mass flux condition

$$
\frac{\rho u}{\rho_{0} u_{0}}=\frac{\gamma^{2}}{\gamma^{2}-1}
$$

is met ( $\rho$ is density, $u$ is fluid velocity, $\gamma$ is the adiabatic index, and the subscript zero denotes the far upstream values), i.e., before the flow undergoes a "self-reversal." The BBS result predicts neither the position nor the nature of the cometary bow shock but shows merely that a shock transition must occur some time before condition (1) is met. These theoretical results sparked a vigorous debate concerning the existence, location, and strength of the cometary bow 
shock, with some advocating either that no shock existed and that the flow was completely smooth (a "bow wave") or that the shock was considerably weaker than that suggested by BBS [Wallis, 1971]. However, more recent detailed numerical simulations (see, for example, Schmidt and Wegmann [1982], Schmidt-Voigt [1988], Ogino et al. [1988], and Omidi and Winske [1987], and see, for example, Galeev [1991] and Cravens [1991] for a summary of these results) of the interaction between the solar wind and cometary atmospheres tend to support the existence of a weak bow shock.

With the recent missions to comets Giacobini-Zinner (GZ) and Halley, considerable light has been shed on both the microphysics and macrophysics of the cometary interaction with the solar wind (see, for example, Ip and Axford [1989] for a general review). Unfortunately, with regard to ascertaining the existence (or nonexistence) of a cometary bow shock, the ICE measurements at GZ were not regarded as very conclusive [Bame et al., 1986], although Smith et al. [1986] did conclude eventually that a weak shock was found. The difficulty in detecting the GZ shock may well have been a consequence of assuming that a cometary shock is necessarily thin, a condition which is unlikely to hold at massloading shocks (see below). On the other hand, observations made during the Halley encounter provide much clearer evidence for the existence of a shock [e.g., Neubauer et al., 1986; Coates et al., 1987a, b, 1990a].

Although the observed decelerated flow regime ahead of the shock appears to be reasonably consistent with the gross predictions of fluid dynamical models, there remain nevertheless two significant unresolved problems, both of which can be addressed within the context of a fluid description. The first concerns the relationship between the Mach number of the mass-loading supersonic flow and the location of the shock, while the second concerns the nature and properties of the embedded bow shock. The former issue has been considered recently by Galeev and Khabibrakhmanov [1990] and Khabibrakhmanov et al. [1991] and will not be addressed in this paper. The latter problem has been investigated by Zank and Oughton [1991] for gasdynamical shocks and by Zank et al. [1991] for parallel MHD shocks (hereinafter papers 1 and 2). In this paper we extend the ideas developed in papers 1 and 2 to more general MHD shock configurations.

The observed structure of the embedded bow shock is very different from the simple gasdynamical subshock picture of a sharp discontinuous transition (see, for example, Figure 2.1 of Ip and Axford [1989]). Instead, observations made during the GZ and Halley encounters reveal that (1) the shock transitions tend to be very broad, for example, the shock thickness on the inbound Halley encounter was found to be $\sim 45,000 \mathrm{~km}$ and that on the outbound encounter $\sim 120,000 \mathrm{~km}$, i.e., the shock is several cometary ion gyroradiì thick [Coates et al., 1987b]; (2) the observed shock structure at the inbound Halley encounter consisted of a series of velocity dips and recoveries [Coates et al., 1987a, $b ;$ Neugebauer et al., 1987a] before the flow settled into its final downstream state (see Figure 3 of Coates et al. [1990a]); and (3) the shock observed on the outbound Halley encounter, although apparently quasi-parallel, possessed an unusually strong rotation of the downstream magnetic field, which led Neubauer et al. [1990] to introduce the description "draping shock."

Clearly, the nature of the cometary shock is poorly understood, although simulations suggest that the shock thickness is due to mass loading of the flow already well upstream of the shock [Omidi and Winske, 1987]. In particular, point 1 above distinguishes the cometary shock from ordinary nonreacting gasdynamical or MHD shocks in that mass loading is important within the shock itself. It was pointed out by Neubauer et al. [1990] and subsequently by Zank and Oughton [1991] that when typical cometary gas production rates and dissociation lifetimes [Krankowsky et al., 1986] are used, the ratio of the newly ionized cometary mass flux injected within the shock to the convected incident mass flux can easily achieve values of $\sim 0.06$. Neubauer et al. [1990] suggested that the Rankine-Hugoniot relations should include a source term to account for the significant mass loading within the body of the shock. They further suggested that mass injection at the shock may provide an explanation for the observed "draping shock" but did not develop this model in any detail. It was pointed out in paper 1 , however, that such a strong rotation of the downstream magnetic field would lead to a significant reduction in the downstream gas pressure, which raised questions concerning the admissibility of certain solutions to the RankineHugoniot (R-H) relations. In general, the simple thermodynamical arguments used in gasdynamics and MHD are inappropriate for complex reacting flows experiencing mass loading [Zank, 1991]. In order to understand gasdynamical shocks subjected to significant mass injection, Lax's formulation of the "entropy condition" [Lax, 1973] was used to isolate the physically relevant solutions of the $\mathrm{R}-\mathrm{H}$ conditions in paper 1. Lax's formulation is employed in this paper to consider the more complicated general MHD shock problem with mass loading. Such an approach is, to the best of our knowledge, the first time that the Lax inequalities have been used in the context of MHD flows. It should be noted that the Lax conditions also serve to pick out the stable solutions of the R-H conditions (see appendix).

Although we have so far discussed mass loading at comets only, it has long been thought that ionization processes, and hence mass loading, controlled the interaction between the solar wind and nonmagnetized or weakly magnetized planets [e.g., Wallis, 1973]. As demonstrated by various missions (Pioneer Venus (to Venus), VEGA 1 and 2 and Giotto (to comet Halley), and Phobos 2 (to Mars)), mass loading is ubiquitous, occurring at both nonmagnetic and slightly magnetic objects possessing an atmosphere. However, since planetary atmospheres are gravitationally bound, unlike their more spectacular cometary cousins, the mass-loading regime tends to be localized in a fairly narrow layer above the planetary surface. As discussed by Breus [1991], the solar wind-neutral planetary atmosphere interaction is most likely to possess cometary characteristics during periods of solar maximum rather than solar minimum (for related discussions, see the review articles of Luhmann [1986] and Luhmann and Brace [1991]).

For the purposes of these investigations, the most important question is whether mass loading occurs upstream of the bow shock. As has been demonstrated by Winske [1986] and Omidi and Winske [1987], the pickup and stochastic acceleration of heavy ions upstream of a planetary or cometary bow shock affects the structure of the shock significantly, in terms of both the magnetic turbulence and shock broadening. The thickness of the shock transition was found to scale of the order of a heavy ion gyroradius, rather than a solar wind proton. Thus for the results of this paper to be 
applicable to planetary bow shocks such as Venus and Mars, it will be under the condition that appreciable mass loading upstream of the bow shock occurs. As noted, this is likely to be during solar maximum only.

In section 2 we present a brief discussion of shock waves in classical nonreacting MHD, using as our basis the shock polar relation given by Cabannes [1970]. This is necessary in order to contrast and compare properly the results of the sections that follow. Since a structure reminiscent of a switch-on shock was observed on the outbound GiottoHalley encounter, we also discuss very briefly some properties of classical switch-on shocks in this section. The appropriate mass-loading MHD shock model is presented in section 3, together with a discussion of the effect of adding a very small quantity of mass to a steady flow. We wish to stress that we are not considering a shock structure problem, as BBS, for example, did, but instead are considering the $\mathrm{R}-\mathrm{H}$ relations of a "thick" shock within which a small quantity of mass is added. The relationship of this work to the shock structure problem has been discussed in detail in paper 1. A completely general mass-loading form of the Hugoniot equation is derived in section 3 , and some of the most important differences between mass-loading and nonreacting classical MHD shocks are elucidated here. The determination of which solutions to the $\mathrm{R}-\mathrm{H}$ conditions correspond to physically admissible mass-loading fronts is investigated in section 4 for parallel and oblique shocks. Two completely new kinds of MHD shocks are presented in section 4, these having no classical MHD analogues. In subsection 4.3 we concentrate on the parameters appropriate to the Giott-Halley encounter and discuss some possible limitations to the Viñas-Scudder technique of determining the shock normal from observations [Viñas and Scudder, 1986]. The conclusion is to be found in section 5 .

\section{Classical MHD Shocks}

In order to facilitate comparison of the classical and cometary shock properties, we present in this section a brief overview of classical MHD shock theory in terms of the shock polar relation given by Cabannes [1970]. Our discussion is based on the R-H relations describing a one-fluid plasma. As usual, these conditions express the conservation of mass, momentum, and energy across a discontinuity, together with the requirement that the normal component of the magnetic field and the tangential component of the electric field be continuous across the shock.

If we consider a perfect gas with adiabatic index $\gamma$, then the $\mathrm{R}-\mathrm{H}$ conditions can be reduced to a single algebraic polynomial (the so-called "shock polar") which relates the upstream and downstream Alfvénic Mach numbers [e.g., Cabannes, 1970, chapter 3]:

$$
\begin{aligned}
\left(M_{A 0}^{2}-M_{A}^{2}\right)\left\{( M _ { A 0 } ^ { 2 } - 1 ) ^ { 2 } \left[M_{A 0}^{2}\left(M_{A 0}^{2}-\frac{\gamma+1}{\gamma-1} M_{A}^{2}\right)\right.\right. \\
\left.+\frac{2 C_{s 0}^{2} / V_{A 0}^{2}}{\gamma-1} M_{A 0}^{2}\right]+M_{A 0}^{2}\left[\left(M_{A 0}^{2}-\frac{\gamma+1}{\gamma-1} M_{A}^{2}\right)\right. \\
\left.\left.+\frac{1}{\gamma-1}\left[2 M_{A 0}^{2}-\gamma\left(M_{A 0}^{2}-M_{A}^{2}\right)\right] M_{A}^{2}\right] \tan ^{2} \theta_{0}\right\} \\
=0 .
\end{aligned}
$$

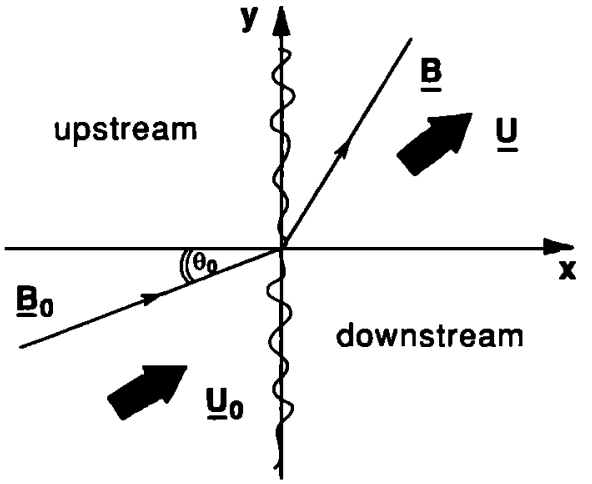

Fig. 1. Schematic of a stationary oblique MHD shock located in the $x=0$ plane of a rectangular Cartesian coordinate system $O X Y Z$. The fluid velocity $u$ and magnetic field $B$ lie in the $(x, y)$ plane, and the electric field $E$ lies along the $z$ axis.

The subscript zero in (2) refers to physical quantities upstream of the shock, and

$$
M_{A}^{2}=\frac{u_{x}^{2}}{V_{A}^{2}}=\frac{u_{x}^{2} \mu \rho}{B_{x}^{2}}
$$

defines the square of the Alfvénic Mach number at any point in the flow. Here $V_{A}$ denotes the component of the Alfvén velocity normal to the shock (see Figure 1 ), $u_{x}$ the flow speed normal to the shock, $B$ the magnetic field, $\rho$ the fluid density, $p$ the gas pressure, and $\mu$ the magnetic permeability. The angle $\theta_{0}$ in (2) is the angle between the magnetic field and the shock normal upstream of the shock and is therefore a measure of the obliquity of the shock (Figure 1). Finally, we use

$$
C_{s 0}=\left(\gamma p_{0} / \rho_{0}\right)^{1 / 2}
$$

to denote the gas sound speed upsteam of the shock. The ratio of sound to Alfvénic speeds in (2) can be rewritten in terms of the plasma beta

$$
\beta_{p}=2 \mu p / B^{2},
$$

as $C_{s 0}^{2} / V_{A 0}^{2}=\frac{1}{2} \gamma \beta_{p 0} \sec ^{2} \theta_{0}$.

The importance of the shock polar relation is that by specifying the upstream variables $\beta_{p 0}, \theta_{0}$, and $M_{A 0}$, the downstream Alfvénic Mach number $M_{A}$ can then be computed easily together with the remaining downstream variables,

$$
\begin{gathered}
B_{y}\left(M_{A}^{2}-1\right)=B_{y 0}\left(M_{A 0}^{2}-1\right) \\
u_{y}=u_{y 0}+\frac{B_{x}}{\mu \rho_{0} u_{x 0}}\left(B_{y 0}-B_{y}\right) \\
p=p_{0}+\rho_{0} u_{x 0}^{2}\left(1-\frac{1}{R}\right)+\frac{1}{2 \mu}\left(B_{y 0}^{2}-B_{y}^{2}\right)
\end{gathered}
$$

Here $R=\rho / \rho_{0}$, the shock compression ratio, is equal to $M_{A 0}^{2} / M_{A}^{2}$.

To elucidate the role of the Alfvenic and magnetosonic modes in classifying MHD shocks, the shock polar (2) can be rewritten as 

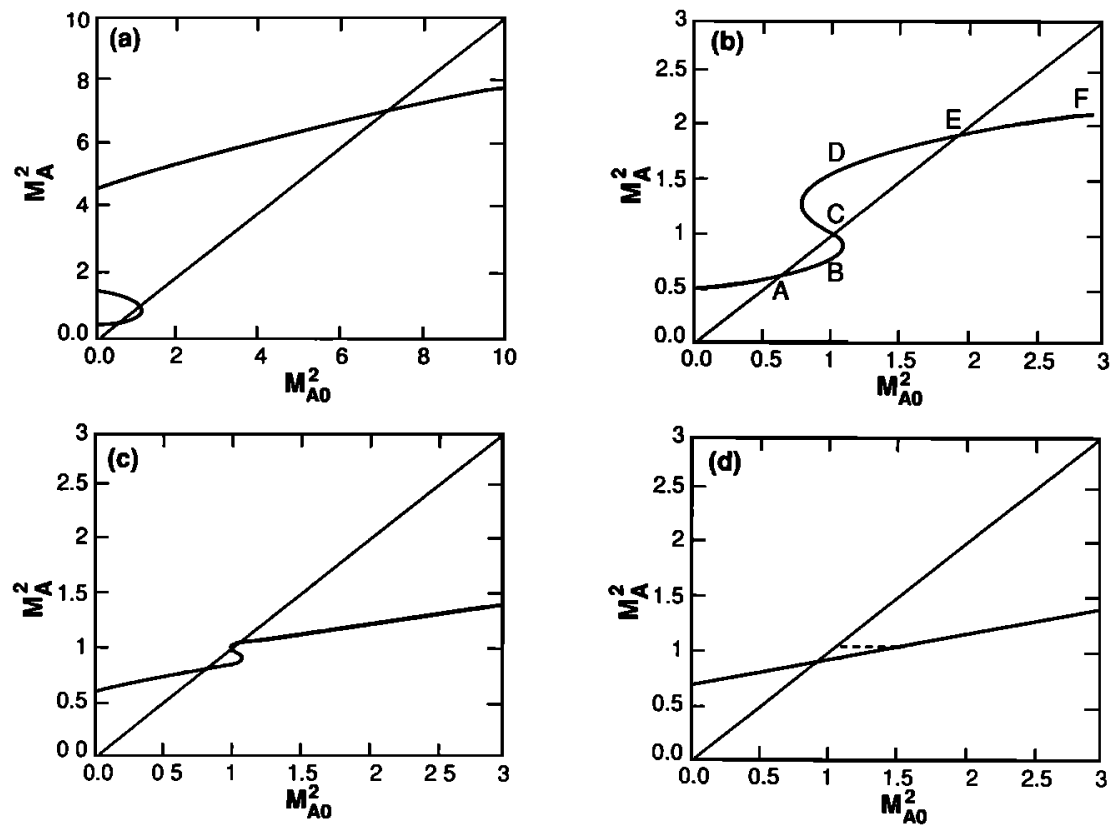

Fig. 2. The change in the Alfvén Mach number shock polar relation of Cabannes [1970] as the upstream magnetic field angle $\theta_{0}$ decreases for shocks in which $\beta_{p 0}=1\left(\theta_{0}=(a) 60^{\circ},(b) 30^{\circ},(c) 5^{\circ}\right.$, and $\left.(d) 0^{\circ}\right)$. Figure $2 d$ illustrates both pure gasdynamical shocks (the solid line) and switch-on shocks (the dashed line).

$$
\begin{aligned}
M_{A 0}^{2}-M_{A}^{2}=2\left(M_{A}^{2}-1\right)\left[M_{A}^{2}-\left(M_{0}^{-}\right)^{2}\right]\left[M_{A}^{2}-\left(M_{0}^{+}\right)^{2}\right] \\
\cdot\left\{(\gamma-1)\left(M_{A}^{2}-1\right)^{2}+\left[1-\left(M_{0}^{-}\right)^{2}\right]\left[\left(M_{0}^{+}\right)^{2}-1\right]\right. \\
\left.\cdot\left[(2-\gamma) M_{A}^{2}+\gamma-1\right]\right\}^{-1},
\end{aligned}
$$

where we have introduced the upstream fast and slow magnetosonic speeds (normalized to the normal upstream Alfvén speed),

$$
M_{0}^{-}=V_{s 0} / V_{A 0} ; \quad M_{0}^{+}=V_{f 0} / V_{A 0},
$$

i.e., $V_{p}= \pm V_{s}, \pm V_{f}$ are the solutions to the magnetosonic dispersion relation

$$
V_{p}^{4}-\left(\frac{\gamma p}{\rho}+\frac{B^{2}}{\mu \rho}\right) V_{p}^{2}+\frac{\gamma p}{\rho} \frac{B_{x}^{2}}{\mu \rho}=0
$$

In general, $0<M_{0}^{-}<1$ and $M_{0}^{+}>1$.

For a shock to be compressive, it is clearly necessary that $M_{A 0}^{2}>M_{A}^{2}$, from which it follows that $M_{A}^{2}$ must satisfy one of the inequalities

$$
\begin{aligned}
& \left(M_{0}^{-}\right)^{2}<M_{A}^{2}<1 ; \\
& M_{A}^{2}>\left(M_{0}^{+}\right)^{2}>1 .
\end{aligned}
$$

If the downstream fluid speed normal to the shock satisfies (10) (i.e., is sub-Alfvénic), then the shock is described as a slow mode shock, whereas normal fluid speeds satisfying (11) are called fast mode shocks. From (7), there exist three solutions for which $M_{A 0}^{2}=M_{A}^{2}$ and for which the compression ratio is equal to unity. These solutions correspond to an Alfvén shock $\left(M_{A}=1\right)$, a fast magnetosonic wave $\left(M_{A}=\right.$ $\left.M_{0}^{+}\right)$, and a slow magnetosonic wave $\left(M_{A}=M_{0}^{-}\right)$.

Examples of the shock polar relation (2) are illustrated in Figure 2 for four different upstream magnetic field angles $\boldsymbol{\theta}_{\mathbf{0}}$ $\left(60^{\circ}, 30^{\circ}, 5^{\circ}, 0^{\circ}\right)$ and a plasma beta $\beta_{p 0}=1$. Two distinct solutions are illustrated in each of the panels of Figure 2: the straight-line solution $M_{A 0}^{2}=M_{A}^{2}$ and the solution to the cubic equation (enclosed by braces in (2)). The requirement that MHD shocks be compressive implies that the only valid shock solutions are those that lie on or beneath the $M_{A 0}^{2}=$ $M_{A}^{2}$ graph. Thus the arcs ABC and EF of Figure $2 b\left(\theta_{0}=\right.$ $30^{\circ}$ ) correspond to compressive solutions. The arc $A B$ represents slow mode shocks while fast mode shocks are located on the curve EF. Points A, C, and E correspond to the slow magnetosonic wave, the Alfvén shock, and the fast magnetosonic mode, respectively. Point $\mathrm{B}$, at which $\boldsymbol{M}_{A 0}=$ 1 , denotes the location, in the $\left(M_{A 0}^{2}, M_{A}^{2}\right)$ phase space, of the (slow mode) switch-off shock (i.e., a shock in which the downstream tangential magnetic field is zero; see Figures 3 and 4).

There is currently renewed debate as to what the arc BC represents, BC being the locus of intermediate shocks [Anderson, 1963]. It has long been thought, on the basis of direct stability considerations [Akhiezer et al., 1959; Cabannes, 1970, chapter 3], that intermediate shocks could not exist, instead disintegrating spontaneously into a slow shock and a rotational discontinuity [Kantorwitz and Petschek, 1966]. However, recent numerical simulations undertaken by $W u$ [1987] have sparked renewed interest in the possible existence and properties of intermediate shocks [Hau and Sonnerup, 1989; Kennel et al., 1989; Freistühler, 1991]. Use of the Lax entropy conditions suggests that at least a portion of the arc BC should admit stable intermediate shocks (see section 4).

For a shock propagating parallel to the upstream magnetic field, $\theta_{0}=0$ (see Figure 2), so that (2) becomes

$$
M_{A}^{2}=\frac{\gamma-1}{\gamma+1} M_{A 0}^{2}+\frac{2}{\gamma+1} \frac{C_{s 0}^{2}}{V_{A 0}^{2}} .
$$




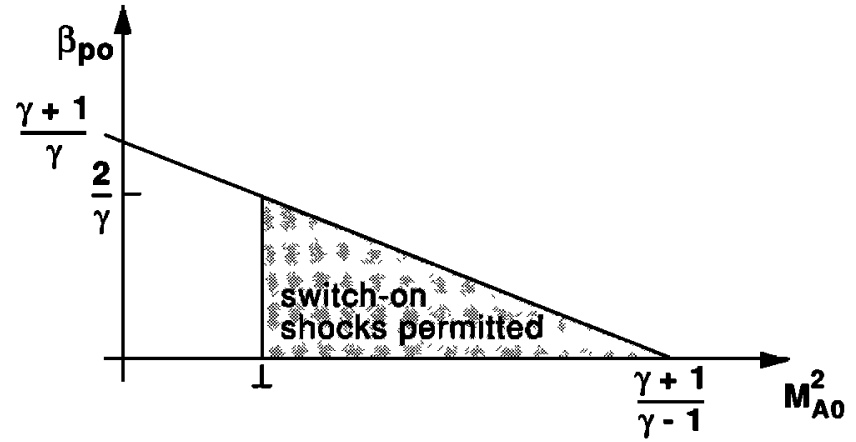

Fig. 3. The parameter regime admitting switch-on shocks for classical nonreacting MHD. Note the controlling role of the adiabatic index $\gamma$ of the plasma in determining the admissible parameter regime.

Thus the shock polar degenerates into a simple straight line, and by dividing both sides of (12) by $M_{A 0}^{2}$, one finds that the inverse compression ratio $\rho_{0} / \rho$ corresponds to that of a pure gas shock [e.g., Landau and Lifshitz, 1979]. To calculate the downstream tangential magnetic field in the simultaneous limits of $\theta_{0} \rightarrow 0$ and $M_{A} \rightarrow 1$, use of (6) to eliminate $\tan \theta_{0}$ in (2) yields

$$
\begin{aligned}
\tan ^{2} \theta=B_{y}^{2} / B_{x}^{2}= & 2\left(M_{A 0}^{2}-1\right) \\
& \cdot\left[1-\frac{\gamma \beta_{p 0}}{2}-\frac{\gamma-1}{2}\left(M_{A 0}^{2}-1\right)\right] .
\end{aligned}
$$

Clearly, for switch-on shocks to exist, it is necessary that

$$
1<M_{A 0}^{2} ; \quad 0<\beta_{p 0}<\frac{\gamma+1}{\gamma}-\frac{\gamma-1}{\gamma} M_{A 0}^{2},
$$

so that we require at least $\beta_{p 0}<2 / \gamma$. However, as illustrated in Figure 3 , the conditions for the existence of a classical MHD switch-on shock are even more stringent when viewed in the $\left(\beta_{p 0}, M_{A 0}^{2}\right)$ parameter space. As is discussed below and in paper 2, however, "switch-on" shocks in massloading flows do not have to satisfy this rather restrictive requirement.

In concluding this brief overview, we plot the downstream tangential magnetic field and the downstream normalized pressure and density as functions of the square of the upstream Alfvénic Mach number in Figure 4 for the illustrative case $\theta_{0}=30^{\circ}, \beta_{p 0}=1$. The rotation of the magnetic field for intermediate shocks is clearly illustrated in Figure $4 a$.

\section{MHD Equations for Mass-Loading Shocks}

The system of equations used to investigate MHD shocks experiencing significant mass loading are appropriate extensions of those given in papers 1 and 2. Following the reasoning employed in paper 1 , we assume that the pickup ion velocity distribution is shell-like due to rapid pitch angle scattering [Neugebauer et al., 1987b, 1989; Coates et al., $1990 \mathrm{~b}$ ]. Thus a common velocity for the plasma components is established, so ensuring that the plasma flow becomes essentially hydrodynamic. Such a description is certainly appropriate to those ions experiencing "turbulent pickup," but the "laminar pickup" of heavy ions (i.e., ion pickup by the large-scale quasi-stationary field) can be described adequately using a kinetic description only [Papadopoulos et al., 1987]. A detailed discussion concerning the suitability of the hydrodynamic description is to be found in the work by Breus and Krymskii [1992] and Tatrallyay et al. [1984]. Further discussion can be found in the reviews of Galeev [1991] and Cravens [1991]. The rapid isotropization of the picked-up heavy ions suggests, incidentally, that one should choose the ratio of specific heats $\gamma$ to be $5 / 3$, appropriate to 3 degrees of freedom, rather than the more commonly used $\gamma=2$ (see also Tatrallyay et al. [1984]). Finally, since the newly ionized particles have a very small initial velocity, they add negligibly to the normal momemtum balance, so we need account for their presence only in the total mass flux equation and the tangential momentum balance equation. As recognized by Axford [1964] and BBS, it is the addition of mass that dominates the solar wind-cometary atmosphere interaction. However, because the addition of mass to the flow destroys the tangential invariance of the fluid equations, it is important to keep track of the tangential momentum and energy contribution from the cometary ions, especially if one introduces a reference frame different from the cometary reference frame [Neubauer et al., 1990; paper 2].

The importance of mass loading is easily inferred from in situ observations. The thickness of the "outbound" Halley shock observed by Giotto in the normal direction is $d=$ $120,000 \mathrm{~km}$. Thus, using the gas production rates of Krankowsky et al. [1986] and a $10^{6}$-s lifetime for the dissociation products of cometary neutral molecules implies an injected mass-loading mass flux within the shock of the order of $1.4 \times 10^{7} \mathrm{amu} /\left(\mathrm{cm}^{2} \mathrm{~s}\right)$. On the other hand, Coates et al. $[1990 a]$ have measured the upstream mass flux to be $2.3 \times$ $10^{8} \mathrm{amu} /\left(\mathrm{cm}^{2} \mathrm{~s}\right)$, giving a significant shock mass-loading to upstream mass flux ratio of $\sim 0.06$. For the inbound Halley shock, the measured shock thickness was found to be
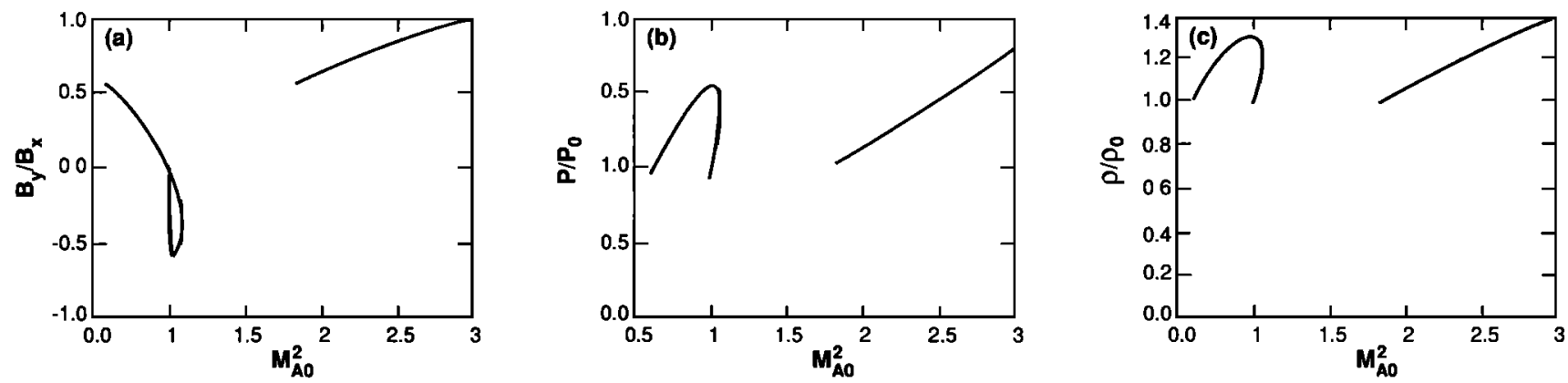

Fig. 4. Plots of $(a)$ the downstream tangential magnetic field, $(b)$ the downstream thermal gas pressure, and (c) downstream density as functions of the square of the incident Alfvén Mach number of the flow for the case $\theta_{0}=30^{\circ}$, $\beta_{p 0}=1$, and $\bar{\alpha}=0$. 


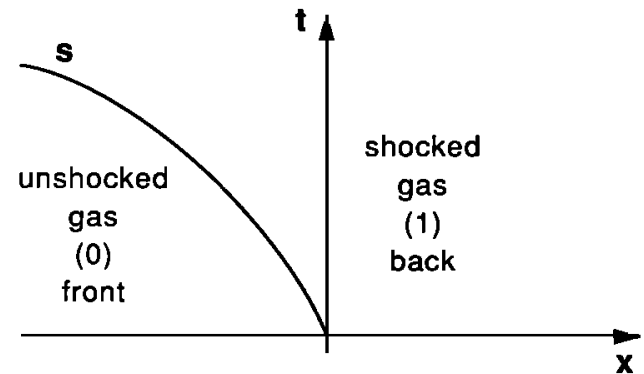

Fig. 5. Sketch of a left-facing shock propagating with speed $s$ in the $(x, t)$ plane.

$\sim 40,000-45,000 \mathrm{~km}$ instead [Coates et al., 1987a, b], which yields a shock mass-loading to upstream mass flux ratio of $\sim 0.005-0.01$. In the calculations below, we adopt a ratio of 0.01 to be conservative.

An idealized magnetohydrodynamical model describing mass injection at some location in the flow can be written down on the basis of the assumptions above. The onedimensional idealized conservation laws are then

$$
\begin{gathered}
\frac{\partial \rho}{\partial t}+\frac{\partial}{\partial x}\left(\rho u_{x}\right)=\alpha \delta(x-s t) \\
\frac{\partial}{\partial t}\left(\rho u_{x}\right)+\frac{\partial}{\partial x}\left(\rho u_{x}^{2}+p+\frac{1}{2 \mu} B^{2}\right)=0 \\
\frac{\partial}{\partial t}\left(\rho \mathbf{u}_{t}\right)+\frac{\partial}{\partial x}\left(\rho u_{x} \mathbf{u}_{t}-\frac{B_{x}}{\mu} \mathbf{B}_{t}\right)=\alpha \mathbf{V}_{t} \delta(x-s t) \\
\frac{\partial}{\partial t}\left(e+\frac{B^{2}}{2 \mu}\right)+\frac{\partial}{\partial x}\left((e+p) u_{x}+\frac{1}{\mu} u_{x} B^{2}-\frac{B_{x}}{\mu} \mathbf{u} \cdot \mathbf{B}\right) \\
=\frac{\alpha}{2} V_{t}^{2} \delta(x-s t) \\
\frac{\partial \mathbf{B}_{t}}{\partial t}+\frac{\partial}{\partial x}\left(u_{x} \mathbf{B}_{t}-B_{x} \mathbf{u}_{t}\right)=0 \\
B_{x}=\text { const, }
\end{gathered}
$$

where $e \equiv \rho u^{2} / 2+\rho \varepsilon$ and $\varepsilon$ is the internal energy of the fluid. The variables have the same definitions as given in the preceding section. Here $\mathbf{u}_{t}=\left(u_{y}, u_{z}\right)$ denotes the transverse velocity field, $B_{t}=\left(B_{y}, B_{z}\right)$, and $V_{t}$ represents the transverse velocity of the newly ionized cometary particles. In addition, we have introduced the "averaged" source term $\alpha \equiv q m_{c} d$, where $q$ is the average production rate of cometary ions, $m_{c}$ the mass of a cometary ion, and $d$ the shock thickness. Finally, $s$ represents the speed of propagation of the shock within which mass loading occurs. For an ideal gas (the case we consider),

$$
\varepsilon=\frac{1}{\gamma-1} \frac{p}{\rho},
$$

so that

$$
e+p=\frac{1}{2} \rho u^{2}+\frac{\gamma}{\gamma-1} p .
$$

The shock configuration is illustrated in Figure 5 for a left-facing shock propagating with velocity $s$.

The suitability of the model (15)-(20) for "real" massloading systems, such as are expected to exist at comets, Venus, and Mars, has been discussed in some detail in paper 1 as well as by Neubauer et al. [1990]. We note simply that our analysis is appropriate provided that (1) onedimensionality is a reasonable approximation and (2) the deceleration of the flow ahead of the shock is reasonably gentle with no steep gradients present. These constraints appear to be supported observationally at Halley, but perhaps less clearly at comet GZ. A discussion of the validity of one-dimensional MHD models is also to be found in the work of Khabibrakhmanov et al. [1991]. We observe merely that an MHD description is valid up until the time when the characteristic spatial and time scales of mass loading are somewhat less than the gyroradius and gyrofrequency of the plasma particles. Since we are not considering a shock structure problem, our MHD description remains valid for the problem at hand. Furthermore, even for the bow shock observed on the outbound Halley encounter, the shock thickness is still sufficiently small when compared to typical gross length scales of the cometary system as to render a one-dimensional shock model reasonably valid.

The conservation laws (15)-(20) can be rewritten in hyperbolic form provided $\Psi=\left(\rho, \mathbf{u}, \mathbf{B}_{t}, p\right)^{t}$ is sufficiently smooth. Thus in the smooth section of the flow,

$$
\frac{\partial \Psi}{\partial t}+A(\Psi) \frac{\partial \Psi}{\partial x}=B(\Psi)
$$

where the matrices $A$ and $B$ are defined to be

$$
\begin{aligned}
& A(\Psi)=\left(\begin{array}{ccccccc}
u_{x} & \rho & 0 & 0 & 0 & 0 & 0 \\
0 & u_{x} & 0 & 0 & \frac{B_{y}}{\mu \rho} & \frac{B_{z}}{\mu \rho} & \frac{1}{\rho} \\
& & & & B_{x} & 0 & 0 \\
0 & 0 & u_{x} & 0 & -\frac{\mu \rho}{\mu \rho} & & \\
& & & & & B_{x} & 0 \\
0 & 0 & 0 & u_{x} & 0 & -\frac{\mu \rho}{\mu} & 0 \\
0 & B_{y} & -B_{x} & 0 & u_{x} & 0 & 0 \\
0 & B_{z} & 0 & -B_{x} & 0 & u_{x} & 0 \\
0 & \gamma p & 0 & 0 & 0 & 0 & u_{x}
\end{array}\right) ; \\
& B(\Psi)=\alpha \delta(x-s t) \\
& \cdot\left(1,-\frac{u_{x}}{\rho},-\frac{u_{y}-V_{y}}{\rho},-\frac{u_{z}-V_{z}}{\rho}, 0,0, \frac{\gamma-1}{2} U^{2}\right)^{t} ; \\
& U^{2}=\left[u_{x}^{2}+\left(u_{y}-V_{y}\right)^{2}+\left(u_{z}-V_{z}\right)^{2}\right],
\end{aligned}
$$

$t$ indicating transpose. The characteristics of (22) are simply those of ordinary MHD [e.g., Cabannes, 1970]:

$C_{0}$

$$
\frac{d x}{d t}=u_{x}
$$

$C_{A, f, s}^{ \pm}$

$$
\frac{d x}{d t}=u_{x} \pm V_{A, f, s},
$$

where $V_{A}=B_{x} /(\mu \rho)^{1 / 2}$ is the Alfvén speed as before and $V_{f}$ and $V_{s}$ are the fast and slow magnetosonic speeds, 


$$
\begin{aligned}
V_{f, s}^{2} \equiv V_{+,-}^{2}=\frac{1}{2} & \left\{\frac{\gamma p}{\rho}+\frac{B^{2}}{\mu \rho}\right. \\
& \left. \pm\left[\left(\frac{\gamma \rho}{\rho}+\frac{B^{2}}{\mu \rho}\right)^{2}-4 \frac{\gamma p}{\rho} \frac{B_{x}^{2}}{\mu \rho}\right]^{1 / 2}\right\}
\end{aligned}
$$

obtained from equation (9).

The importance of the characteristics in deciding whether solutions to the $\mathrm{R}-\mathrm{H}$ conditions correspond to physically realizable shock solutions has been discussed in considerable detail in paper 1 . In summary, use of the characteristics and a geometrical entropy condition [ $\operatorname{Lax}, 1973]$ allow one to avoid using thermodynamical arguments to decide on the physical admissibility of solutions to the R-H conditions. While both the geometrical and thermodynamical entropy conditions yield identical results for nonreacting gasdynamics and MHD, the additional thermodynamical complications introduced through mass loading make thermodynamical criteria completely infeasible to apply [see Zank, 1991].

In the frame of the shock (i.e., $s=0$ ), the jump conditions across a mass-loading front are

$$
\begin{gathered}
{\left[\rho u_{x}\right] \equiv \rho u_{x}-\rho_{0} u_{x 0}=\alpha} \\
{\left[\rho u_{x}^{2}+p+\frac{1}{2 \mu} B_{t}^{2}\right]=0} \\
{\left[\rho u_{x} \mathbf{u}_{t}-\frac{B_{x}}{\mu} \mathbf{B}_{t}\right]=\alpha \mathbf{V}_{t}} \\
{\left[(e+p) u_{x}+\frac{1}{\mu} u_{x} B^{2}-\frac{B_{x}}{\mu} \mathbf{u} \cdot \mathbf{B}\right]=\frac{\alpha}{2} V_{y}^{2} ;} \\
{\left[B_{x}\right]=0 ; \quad\left[u_{x} \mathbf{B}_{t}\right]=B_{x}\left[\mathbf{u}_{t}\right],}
\end{gathered}
$$

which are similar to those given in paper 2 and by Neubauer et al. [1990].

A useful simplification is to work in the normal incidence frame of the flow,

$$
\overline{\mathbf{u}}_{t}=\mathbf{u}_{t}-\mathbf{u}_{t 0}, \quad \overline{\mathbf{v}}_{t}=\mathbf{V}_{t}-\mathbf{u}_{t 0},
$$

so that $\overline{\mathbf{u}}_{t 0}=0$ and $\overline{\mathbf{V}}_{t}$ can be positive, negative, or zero. The R-H conditions above are effectively unchanged except that we now use $\overline{\mathbf{u}}_{t}$ and $\overline{\mathbf{V}}_{t}$ instead. For notational convenience we henceforth omit the bars. It is important to recognize that the additional parameter $V_{t}$ can be of considerable importance because of the translation and should not therefore be neglected. This was a point emphasized by Neubauer et al. [1990], although they chose to work in the de HoffmannTeller frame. The importance of the $V$, term is a consequence of the R-H conditions no longer being invariant with respect to tangential flows.

We can also choose $B_{z 0}=0$ without loss of generality (which implies that $u_{z 0}=0$ anyway and so $\bar{V}_{z}=V_{z}$ ). In this case, the $z$ component of the tangential momentum condition (28) reduces to

$$
\left(M_{A}^{2}-1\right) \frac{B_{z}}{B_{x}}=\frac{\alpha}{\rho_{0} u_{x 0}} M_{A 0}^{2} \frac{V_{z}}{u_{x 0}},
$$

where use has been made of (30). Clearly, if the coplanarity theorem is to hold, we need $\left|M_{A 0}^{2} V_{z} / u_{x 0}\right| \ll 1$. However, Neubauer et al. [1990] found that observations of the magnetic field at the outbound Halley shock suggest that the coplanarity theorem is valid because the observed upstream and downstream magnetic field vectors fulfill a condition sufficient for coplanarity given in that paper (p. 469, left center). On considering the entire shock surface, the sufficient condition is fulfilled only on the intersection $I$ between the shock surface and the plane through the cometary nucleus spanned by the upstream solar wind vector and the magnetic field vector. The sufficient condition for coplanarity given by Neubauer et al. [1990] will, however, no longer be valid far from the plane described above, so our analysis applies only to that part of the shock surface reasonably close to $I$. We will assume that the shock is coplanar and thus significantly reduce the algebraic labor.

\subsection{Mass Addition and Steady Flows}

Insight into the effect of adding a small quantity of mass, transverse momentum, and energy to a perfect, steadily flowing gas is gained by considering small-amplitude perturbations to the R-H conditions (26)-(30). After linearization we obtain the small-amplitude relations (i.e., after using $\Psi=$ $\Psi_{0}+\delta \Psi$, and assuming that $\left.\alpha \ll \rho_{0} u_{x 0}, V_{y} \ll u_{x 0}\right)$

$$
\begin{gathered}
u_{x 0} \delta \rho=\alpha-\rho_{0} \delta u_{x} \\
\delta p+\frac{B_{y 0}}{\mu} \delta B_{y}=-\alpha u_{x 0}-\rho_{0} u_{x 0} \delta u_{x} \\
\delta u_{y}=\frac{B_{x}}{\mu \rho_{0} u_{x 0}} \delta B_{y}+\frac{\alpha}{\rho_{0} u_{x 0}} V_{y} \\
u_{x 0} \delta B_{y}+B_{y 0} \delta u_{x}-B_{x} \delta u_{y}=0
\end{gathered}
$$

The changes in the transverse magnetic field and flow velocity and the pressure can all be expressed in terms of $\delta u_{x}$ and the loading terms. Thus

$$
\begin{array}{r}
\frac{\delta B_{y}}{B_{x}}=-\frac{M_{A 0}^{2} \tan \theta_{0}}{M_{A 0}^{2}-1} \frac{\delta u_{x}}{u_{x 0}}+\frac{\alpha V_{y}}{\rho_{0} u_{x 0}^{2}} \frac{M_{A 0}^{2}}{M_{A 0}^{2}-1} \\
\delta u_{y}=-\frac{\tan \theta_{0}}{M_{A 0}^{2}-1} \delta u_{x}+\frac{\alpha V_{y}}{\rho_{0} u_{x 0}} \frac{M_{A 0}^{2}}{M_{A 0}^{2}-1} ; \\
\delta p=-\rho_{0} u_{x 0}\left(1-\frac{\tan ^{2} \theta_{0}}{M_{A 0}^{2}-1}\right) \delta u_{x} \\
-\alpha u_{x 0}\left(1+\frac{\tan \theta_{0}}{M_{A 0}^{2}-1} \frac{V_{y}}{u_{x 0}}\right) .
\end{array}
$$

Turning now to the total energy equation (29), linearization and use of (34)-(36) yields

$$
\begin{aligned}
& -\frac{1}{\gamma-1} u_{x 0}^{2} \delta u_{x}+\frac{1}{\gamma-1} C_{s 0}^{2} \delta u_{x} \\
& \quad+\frac{1}{\gamma-1} V_{A 0}^{2} \tan ^{2} \theta_{0} \frac{M_{A 0}^{2}}{M_{A 0}^{2}-1} \delta u_{x} \\
& =\frac{\gamma+1}{2(\gamma-1)} \frac{\alpha u_{x 0}^{2}}{\rho_{0}}+\frac{\alpha}{\rho_{0}} V_{y}^{2}+\frac{1}{\gamma-1} \frac{u_{x 0}^{2} \tan \theta_{0}}{M_{A 0}^{2}-1} \frac{\alpha V_{y}}{\rho_{0} u_{x 0}} .
\end{aligned}
$$

Some simple manipulations lead to 


$$
\begin{aligned}
& -\frac{1}{\gamma-1} \frac{1}{u_{x 0}^{2}-V_{A 0}^{2}}\left[u_{x 0}^{4}-u_{x 0}^{2}\left(C_{s 0}^{2}+V_{A 0}^{2} \sec ^{2} \theta_{0}\right)\right. \\
& \left.+C_{s 0}^{2} V_{A 0}^{2}\right] \delta u_{x}=\frac{1}{2} \frac{\gamma+1}{(\gamma-1)} \frac{\alpha u_{x 0}^{2}}{\rho_{0}}+\frac{\alpha}{\rho_{0}} V_{y}^{2} \\
& +\frac{1}{\gamma-1} \frac{u_{x 0}^{2} \tan \theta_{0}}{M_{A 0}^{2}-1} \frac{\alpha V_{y}}{\rho_{0} u_{x 0}},
\end{aligned}
$$

and the term in square brackets corresponds to the usual magnetosonic dispersion relation (9). Thus the change in the normal velocity component $u_{x}$ after mass, transverse momentum, and energy addition is given by

$$
\begin{aligned}
\delta u_{x}=\frac{-\left(u_{x 0}^{2}-V_{A 0}^{2}\right)}{\left(u_{x 0}^{2}-V_{f 0}^{2}\right)\left(u_{x 0}^{2}-V_{s 0}^{2}\right)}\left[\frac{\gamma+1}{2} \frac{\alpha u_{x 0}^{2}}{\rho_{0}}\right. \\
\left.+(\gamma-1) \frac{\alpha V_{y}^{2}}{\rho_{0}}+\frac{u_{x 0}^{2} \tan \theta_{0}}{M_{A 0}^{2}-1} \frac{\alpha V_{y}}{\rho_{0} u_{x 0}}\right],
\end{aligned}
$$

where $V_{f}$ and $V_{s}$ denote the fast and slow magnetosonic speeds, respectively. The first term in the square brackets represents the mass-loading contribution, the second corresponds to the addition of energy, and the final term corresponds to the deposition of transverse momentum.

Consider the effect of mass loading and energy addition alone on $\delta u_{x}$. Since $V_{s 0}^{2}<V_{A 0}^{2}<V_{f 0}^{2}$, truly subsonic flows upstream of the loading region, i.e., flows satisfying $u_{x 0}<$ $V_{s 0}$, imply $\delta u_{x}>0$. In other words, mass loading of a sub(slow) magnetosonic flow accelerates the flow. Conversely, for flows satisfying either $u_{x 0}>V_{f 0}$ or $V_{s 0}<$ $u_{x 0}<V_{A 0}$, we have $\delta u_{x}<0$; i.e., mass loading of supersonic flows decelerates the flow, as is well known. On the other hand, somewhat surprisingly, for flow velocities intermediate between the Alfvénic and fast magnetosonic speeds, $\delta u_{x}>0$, thus leading to an increase in the flow velocity.

The effect of mass loading on weak MHD shocks can be inferred from the classical shock polar plots (Figure 2). The straight-line solutions of the classical shock polar relation $M_{A}^{2}=M_{A 0}^{2}$ correspond to the steady flows discussed above, as do the weak slow mode (point $A$ of Figure $2 b$ ), weak intermediate (point $\mathrm{C}$ ), and weak fast mode (E) shocks. From (37) the line segment joining the origin to A in Figure $2 b$ must be displaced upward after mass loading whereas that from $A$ to $C$ will be displaced downward. What was the slow magnetosonic point $A$ will now be a "singular point" on the perturbed shock polar and no longer correspond to a feasible flow solution linking the upstream and downstream states. The line segment $C E$ is displaced upward after mass loading whereas EF is displaced downward, thereby making the fast magnetosonic speed "singular" in the sense described above. It is evident that mass loading can split the shock polar curve into as many as three disjoint curves, and this is indeed confirmed numerically below.

\subsection{Derivation of the Hugoniot Equation}

In this section we derive the generalized form of the mass-loading Hugoniot equation, discuss some of its properties in relation to the Rayleigh curves, and discuss a modified form of the shock polar relation. Let $m=\rho_{0} u_{x 0}$, and introduce the specific volume $\tau=1 / \rho$. Then we have

$$
\begin{aligned}
& u_{x 0}=m \tau_{0} ; \\
& u_{x 1}=m(1+\bar{\alpha}) \tau_{1} \equiv m \bar{\tau}_{1},
\end{aligned}
$$

where $\bar{\alpha}=\alpha / m$ and the subscript 1 denotes the downstream value of the fluid variable. From the momentum equation (27) we obtain

$$
m^{2}=\frac{[p]+\left[B_{y}^{2}\right] / 2 \mu}{\tau_{0}-(1+\bar{\alpha}) \bar{\tau}_{1}} .
$$

It is convenient to introduce a slightly different form of the jump relation, defined by

$$
\{\bar{a} b\} \equiv \bar{a}_{1} b_{1}-a_{0} b_{0}=(1+\bar{\alpha}) a_{1} b_{1}-a_{0} b_{0},
$$

which tends to simplify some of the algebraic manipulations below.

By means of (38)-(40) the total energy equation (29) can be reduced to

$$
\begin{array}{r}
\{\bar{\epsilon}\}+\{\bar{\tau} p\}-\frac{1}{2}\left[p+\frac{1}{2 \mu} B_{y}^{2}\right]\left(\bar{\tau}_{1}+\tau_{0}\right)-\frac{\bar{\alpha}}{2} m^{2} \tau_{0} \bar{\tau}_{1} \\
+\frac{1}{\mu}\left\{\bar{\tau} B_{y}^{2}\right\}+\frac{1}{2}\left\{\bar{u}_{y}^{2}\right\}-\frac{B_{x}}{\mu m}\left[u_{y} B_{y}\right]=\frac{\bar{\alpha}}{2} V_{y}^{2},
\end{array}
$$

with $\varepsilon$ the internal energy of the fluid. Repeated use of the transverse momentum relation (28) further reduces (41) to

$$
\begin{aligned}
\{\bar{\epsilon}\}+\frac{1}{2}\langle p\rangle\{\bar{\tau}\}- & \frac{1}{4 \mu}\left(\bar{\tau}_{1}+\tau_{0}\right)\left[B_{y}^{2}\right]+\frac{1}{\mu}\left\{\bar{\tau} B_{y}^{2}\right\} \\
& -\frac{1}{2} \frac{1}{1+\bar{\alpha}} \frac{B_{x}^{2}}{\mu^{2} m^{2}}\left[B_{y}\right]\left(B_{y}\right)-\frac{1}{2} \bar{\alpha} m^{2} \tau_{0} \bar{\tau}_{1} \\
& -\frac{\bar{\alpha}}{1+\bar{\alpha}} \frac{B_{x}}{\mu m} B_{y 0} V_{y}=\frac{1}{2} \frac{\bar{\alpha}}{1+\bar{\alpha}} V_{y}^{2}
\end{aligned}
$$

where the angle brackets are defined to be $\langle Q\rangle=Q_{1}+Q_{0}$ as usual. If use is made of the result

$$
\begin{array}{r}
-\frac{1}{4}\left(\bar{\tau}_{1}+\tau_{0}\right)\left[B_{y}^{2}\right]+\left\{\bar{\tau} B_{y}^{2}\right\}-\frac{1}{2} \frac{1}{1+\bar{\alpha}}\left\{\bar{\tau} B_{y}\right\}\left\langle B_{y}\right\rangle=\frac{1}{4}\{\bar{\tau}\}\left[B_{y}\right]^{2} \\
+\frac{1}{2} \frac{\bar{\alpha}}{1+\bar{\alpha}}\left\{\bar{\tau} B_{y}\right\}\left\langle B_{y}\right\rangle,
\end{array}
$$

then (42) reduces eventually to the mass-loading form of the Hugoniot function

$$
\begin{aligned}
\mathscr{H}(\bar{\tau}, p)=\{\bar{\varepsilon}\} & +\frac{1}{2}\langle p\rangle\{\bar{\tau}\}+\frac{1}{4 \mu}\{\bar{\tau}\}\left[B_{y}\right]^{2}+\frac{\bar{\alpha}}{2} m^{2} \tau_{0} \bar{\tau} \\
& +\frac{1}{2} \frac{\bar{\alpha}}{1+\bar{\alpha}} \frac{B_{x}}{\mu m}\left[B_{y}\right] V_{y}-\frac{1}{2} \frac{\bar{\alpha}}{1+\bar{\alpha}} V_{y}^{2},
\end{aligned}
$$

and the Hugoniot equation is $\mathscr{H}\left(\bar{\tau}_{1}, p_{1}\right)=0$. The Hugoniot function (43) is, in many respects, very similar to the Hugoniot of classical MHD [see Cabannes, 1970, p. 55] except that the jump condition \{\} contains the effect of 
mass loading. The fourth term in (43) is identical to that obtained already in paper 1 for the normal gasdynamical case, and the remaining two terms arise as a consequence of the lack of tangential invariance in oblique mass-loading flows. In fact, the last term of (43) is present in the oblique gasdynamical Hugoniot as well. As described in paper 1, the lack of tangential invariance results from mass loading within the shock introducing shearing stresses into the flow, something which distinguishes mass-loading shocks from both nonreacting shocks and combustion shocks.

The mass-loading MHD Hugoniot (43) is a function of the upstream Mach number through $m^{2}$ since $M_{0}^{2} \equiv u_{x 0}^{2} / C_{s 0}^{2} \equiv$ $u_{x 0}^{2} / \gamma p_{0} \tau_{0}$ for an ideal gas. Thus even though $\bar{\alpha}$ is a rather small term, its associated momentum contribution in the shock frame can be significant.

The expression for $B_{y}$ in the Hugoniot (43) can be written in terms of the inverse compression ratio $\tau$ by means of (28) and (30). Thus

$$
\left[B_{y}\right]=\frac{\tau_{0}-\bar{\tau}}{\bar{\tau}-\tau_{*}} B_{y 0}+\frac{\bar{\alpha}}{1+\bar{\alpha}} \frac{B_{x}}{m} \frac{V_{y}}{\bar{\tau}-\tau_{*}},
$$

where we have introduced

$$
\tau_{*} \equiv \frac{1}{1+\bar{\alpha}} \frac{B_{x}^{2}}{\mu \rho_{0}^{2} u_{x 0}^{2}}=\frac{\tau_{0}}{1+\bar{\alpha}} \frac{1}{M_{A 0}^{2}} .
$$

The effect of shearing on the magnetic field, induced by mass loading within the shock, is evident from (44) and merely illustrates that the sheared flow drags the magnetic field within the shock. Even for a parallel shock, for which $B_{y 0}=$ 0 , the downstream magnetic field is still rotated since

$$
\frac{B_{y}}{B_{x}}=\frac{\bar{\alpha}}{1+\bar{\alpha}} \frac{\tau_{0}}{\bar{\tau}-\tau_{*}} \frac{V_{y}}{u_{x 0}} .
$$

Thus mass loading will always "switch a shock on" regardless of the magnitude of the plasma beta, and this represents yet another important difference between reacting and nonreacting MHD shocks. It is shown below that the singularity associated with (46) never becomes particularly important because $\bar{\tau} \not \tau_{*}$ if we insist that the thermal pressure $p$ be strictly positive.

From (46) the Hugoniot function for parallel shocks is given by

$$
\begin{aligned}
& \mathscr{H}(\bar{\tau}, p)=\{\bar{\varepsilon}\}+\frac{1}{2}\langle p\rangle\{\bar{\tau}\}+\frac{\tau_{0}^{2} B_{x}^{2}}{4 \mu}\{\bar{\tau}\}\left(\frac{\bar{\alpha}}{1+\bar{\alpha}}\right)^{2} \\
& \cdot\left(\frac{V_{y} / u_{x 0}}{\bar{\tau}-\tau_{*}}\right)^{2}-\frac{\bar{\alpha}}{2} m_{2} \tau_{0} \bar{\tau}+\frac{\bar{\alpha}}{1+\bar{\alpha}} \frac{\tau_{0}^{2} B_{x}^{2}}{2 \mu} \\
& \cdot\left(\frac{\bar{\alpha}}{1+\bar{\alpha}}\right)^{2}\left(\frac{V_{y} / u_{x 0}}{\bar{\tau}-\tau_{*}}\right)^{2}-\frac{1}{2} \frac{\bar{\alpha}}{1+\bar{\alpha}} V_{y}^{2} .
\end{aligned}
$$

Similarly, for a perpendicular MHD shock configuration, $B_{x}=0$ implies $\tau_{*}=0$ and thus $B_{y}=\tau_{0} B_{y} / \bar{\tau}$. Hence the Hugoniot for perpendicular mass-loading shocks reduces to

$$
\begin{array}{r}
\mathscr{H}(\bar{\tau}, p)=\{\bar{\varepsilon}\}+\frac{1}{2}\langle p\rangle\{\bar{\tau}\}+\frac{B_{y 0}^{2}}{4 \mu} \frac{\{\bar{\tau}\}^{3}}{\bar{\tau}^{2}}-\frac{\bar{\alpha}}{2} m^{2} \tau_{0} \bar{\tau} \\
-\frac{1}{2} \frac{\bar{\alpha}}{1+\bar{\alpha}} V_{y}^{2}
\end{array}
$$

This relation is similar to the oblique mass-loading gasdynamical Hugoniot, except for the $\{\bar{\tau}\}^{3}$ term. The Hugoniot (48) is nevertheless somewhat different from the usual perpendicular form of the Hugoniot for classical nonreacting MHD.

For an ideal gas the Hugoniot (43) can be rearranged as

$$
\begin{aligned}
2 \Gamma \mathscr{H}(\bar{\tau}, p)= & \left(\bar{\tau}-\Gamma \tau_{0}\right) p-\left(\tau_{0}-\Gamma \bar{\tau}\right) p_{0}+\frac{\Gamma}{2 \mu}\{\bar{\tau}\}\left[B_{y}\right]^{2} \\
& -\bar{\alpha} \Gamma m^{2} \tau_{0} \bar{\tau}+\frac{\bar{\alpha}}{1+\bar{\alpha}} \Gamma \frac{\tau_{0} B_{x}}{\mu} \frac{V_{y}}{u_{x 0}}\left[B_{y}\right] \\
& -\frac{\bar{\alpha}}{1+\bar{\alpha}} \Gamma V_{y}^{2},
\end{aligned}
$$

where $\Gamma \equiv(\gamma-1) /(\gamma+1)$. Unlike nonreacting MHD, equations (49) and (44) reveal that a state cannot be connected to itself by a mass-loading front since $\mathscr{H}\left(\bar{\tau}_{0}, p_{0}\right) \neq 0$. As shown explicitly by Zank [1991], it is the fact that $\left(\bar{\tau}_{0}, p_{0}\right)$ does not, in general, lie on the Hugoniot curve that renders the notion of "physical entropy" ineffective in determining the physically relevant $\mathrm{R}-\mathrm{H}$ solutions. This is because one needs further thermodynamical information to ascertain whether $\mathscr{H}$ possesses a turning point on the orbit of a particular set of integral curves which join the upstream and downstream states. For complicated reacting flows, such additional thermodynamical constraints are not easily established. This then is the motivation behind using a geometric formulation of the "entropy condition" to isolate the physically relevant solutions to the R-H conditions. For a given $\bar{\alpha}$ and $V_{y}, \mathscr{H}(\bar{\tau}, p)=0$ is the locus of all possible states that can be connected to the given state $\left(\tau_{0}, p_{0}\right)$.

Let us introduce the notation

$$
\begin{gathered}
\tilde{p}=\frac{p}{p_{0}}, \quad \bar{\tau}=\frac{\bar{\tau}}{\tau_{0}}, \quad \tilde{\tau}_{*}=\frac{\tau_{*}}{\tau_{0}}, \\
\tilde{B}_{y}=\frac{B_{y}}{B_{x}}, \quad \tilde{V}_{y}=\frac{V_{y}}{u_{x 0}},
\end{gathered}
$$

since then $\mathscr{H}(\bar{\tau}, p)=0$ reduces to

$$
\begin{aligned}
\frac{p}{p_{0}}= & \frac{1-\Gamma \bar{\tau}}{\bar{\tau}-\Gamma}-\frac{\Gamma}{\beta_{p 0}} \frac{(\bar{\tau}-1)\left(\widetilde{B}_{y}-\tan \theta_{0}\right)^{2}}{\tilde{\tau}-\Gamma} \cos ^{2} \theta_{0} \\
& +\frac{2 \bar{\alpha}}{1+\bar{\alpha}} \frac{\Gamma}{\beta_{p 0}} \frac{1 / \bar{\tau}_{*}}{\bar{\tau}-\Gamma} \cos ^{2} \theta_{0}\left[\tilde{\tau}+\frac{1}{1+\bar{\alpha}}\left[\bar{V}_{y}-(1\right.\right. \\
& \left.\left.+\bar{\alpha}) \tilde{\tau}_{*}\left(\tilde{B}_{y}-\tan \theta_{0}\right)\right] \bar{V}_{y}\right]
\end{aligned}
$$

Also, (44) can be expressed as

$$
\tilde{B}_{y}=\frac{1-\bar{\tau}_{*}}{\bar{\tau}-\bar{\tau}_{*}} \tan \theta_{0}+\frac{\bar{\alpha}}{1+\bar{\alpha}} \frac{\bar{V}_{y}}{\bar{\tau}-\bar{\tau}_{*}} .
$$

The ideal mass-loading MHD Hugoniot possesses essentially the same singularities as those of the nonreacting MHD Hugoniot, namely

$$
\tilde{\tau}=\Gamma \quad \text { and } \quad \tilde{\tau}=\tilde{\tau}_{*},
$$


except that they are now shifted by the mass-loading term $(1+\bar{\alpha})$. However, the line to which the Hugoniot is asymptotic as $\bar{\tau} \rightarrow \infty$ is now rather different, in that we have

$$
\frac{p}{p_{0}}=-\Gamma\left(1+\frac{\sin ^{2} \theta_{0}}{\beta_{p 0}}-\frac{2 \bar{\alpha}}{1+\bar{\alpha}} \frac{\cos ^{2} \theta_{0}}{\beta_{p 0}} \frac{1}{\tilde{\tau}_{*}}\right) .
$$

Thus, depending on the Alfvenic Mach number of the upstream flow and also on the obliquity of the shock, the asymptote (54) can lie in either the $p>0$ or the $p<0$ half plane. Furthermore, the initial state $\left(\tau_{0}, p_{0}\right)$ can also lie on, above, or below the Hugoniot curve, depending on the value of $M_{A 0}^{2}$.

As with the mass-loading gasdynamical Hugoniot, not all points on the mass-loading MHD Hugoniot correspond to physically sensible downstream states. To see this, observe that (39) can be rewritten in terms of the normalized variables as

$$
\begin{aligned}
\frac{2}{\beta_{p 0}} M_{A 0}^{2} \cos ^{2} \theta_{0}= & \frac{\tilde{p}-1}{1-(1+\bar{\alpha}) \tilde{\tau}} \\
& +\frac{1}{\beta_{p 0}} \frac{\widetilde{B}_{y}^{2}-\tan ^{2} \theta_{0}}{1-(1+\bar{\alpha}) \bar{\tau}} \cos ^{2} \theta_{0} .
\end{aligned}
$$

Sensible solutions of the $\mathbf{R}-\mathbf{H}$ conditions are obviously restricted to those downstream $\bar{\tau}$ and $\tilde{p}$ for which the right-hand side of (55) is positive. For parallel shocks we are therefore restricted to those segments of the Hugoniot which lie within the following regions of the $(\tilde{\tau}, \tilde{p})$ plane:

$$
\bar{\tau}<(>) \frac{1}{1+\bar{\alpha}} \quad \tilde{p}>(<) 1-\frac{1}{\beta_{p 0}}\left(\frac{\bar{\alpha}}{1+\bar{\alpha}}\right)^{2} \frac{\tilde{V}_{y}^{2}}{\left(\tilde{\tau}-\tilde{\tau}_{*}\right)^{2}} .
$$

More generally, for arbitrarily oblique shocks we are restricted to the following regimes:

$$
\begin{gathered}
\tilde{\tau}<(>) \frac{1}{1+\bar{\alpha}} \\
\tilde{p}>(<) 1-\frac{1}{\beta_{p 0}}\left(\widetilde{B}_{y}^{2}-\tan ^{2} \theta_{0}\right) \cos ^{2} \theta_{0},
\end{gathered}
$$

of the $(\tilde{\tau}, \tilde{p})$ plane.

Solutions to the $\mathrm{R}-\mathrm{H}$ conditions can be obtained by solving (51) simultaneously with the total momentum equation

$$
\begin{aligned}
\frac{p}{p_{0}}=\left(1+\frac{2}{\beta_{p 0}}\right. & \left.\frac{1}{1+\bar{\alpha}} \frac{\cos ^{2} \theta_{0}}{\tilde{\tau}_{*}}+\frac{1}{\beta_{p 0}} \sin ^{2} \theta_{0}\right) \\
& -\frac{1}{\beta_{p 0}} \bar{B}_{y}^{2} \cos ^{2} \theta_{0}-\frac{2}{\beta_{p 0}} \frac{\tilde{\tau}}{\tilde{\tau}_{*}} \cos ^{2} \theta_{0} .
\end{aligned}
$$

Equation (58) is a generalized form of the well-known Rayleigh curve of classical MHD, but unlike nonreacting MHD the curves described by equations (51) and (58) need not intersect at all.

To proceed further, we need to know two things. First, we need to determine which sections of the Hugoniot correspond to submagnetosonic or supermagnetosonic/Alfvénic downstream flows, and second, we need to understand the circumstances under which the Rayleigh curve and the
Hugoniot intersect. As we show below, both these questions are answered by determining the condition under which the Rayleigh line and the Hugoniot touch; i.e., we derive a touching condition at which the tangents of both curves coincide. We need consider only (58) together with (52). Then differentiation of (58) with respect to $\bar{\tau}$ yields

$$
\frac{\partial}{\partial \bar{\tau}}\left(\frac{p}{p_{0}}\right)=-\frac{2}{\beta_{p 0}} \frac{\cos ^{2} \theta_{0}}{\tilde{\tau}_{*}}-\frac{2}{\beta_{p 0}} \tilde{B}_{y} \cos ^{2} \theta_{0}\left(\frac{\partial \tilde{B_{y}}}{\partial \tilde{\tau}}\right),
$$

and we also have

$$
\frac{\partial \tilde{B}_{y}}{\partial \tilde{\tau}}=-\frac{\tilde{B}_{y}}{\tilde{\tau}-\tilde{\tau}_{*}}
$$

after using (52). On combining (59) and (60), we find

$$
\frac{\partial p}{\partial \tau}=-(1+\bar{\alpha})^{2} m^{2}+(1+\bar{\alpha}) \frac{1}{\mu} \frac{B_{y}^{2}}{\bar{\tau}-\tau_{*}} .
$$

Use of the identity

$$
\tau_{*}=\frac{1}{1+\bar{\alpha}} \frac{B_{x}^{2}}{\mu \rho_{0}^{2} u_{x 0}^{2}}=(1+\bar{\alpha}) \frac{B_{x}^{2}}{\mu \rho^{2} u_{x}^{2}}
$$

enables us to recast (61) in the equivalent form

$$
u_{x}^{4}-\left[\left(\frac{\partial p}{\partial \rho}\right)_{S}+\frac{B^{2}}{\mu \rho}\right] u_{x}^{2}+\left(\frac{\partial p}{\partial \rho}\right)_{S} \frac{B_{x}^{2}}{\mu \rho}=0,
$$

where we have assumed constant entropy downstream of the shock. Quite clearly, (62) is exactly equivalent to the magnetosonic dispersion relation (9). Thus, quite remarkably, when the Rayleigh curve is tangent to the Hugoniot, the downstream normal fluid velocity $u_{x}$ is exactly equal to either the fast or the slow magnetosonic speed downstream of the shock. The distinguished points of tangency are analogous to the Chapman-Jouguet points of combustion theory, and they, together with (57) and (55), enable us to classify the nature of the Hugoniot, and hence of the R-H solutions, precisely.

To illustrate the "topological features" of the Hugoniot, we have plotted an illustrative example in Figures $6 a$ and $6 b$, which are appropriate to a parallel shock with an upstream plasma beta $\beta_{p 0}=1$ and $\tilde{V}_{y}=4$. For easy reference we collect together the various results on the figure itself. The shaded regions are bounded by the curves (56) and the appropriate axes. It can be seen that the section of the Hugoniot labeled MN lies outside the shaded area and this segment admits solutions to the $\mathrm{R}-\mathrm{H}$ conditions for which the square of the upstream mass flux, $m^{2}$, is $<0$. Since $\tilde{\tau}=$ $u_{x} / u_{x 0}$, the region to the right of $\bar{\tau}=1$ corresponds to accelerated solutions, and that to the left to decelerated solutions. Note that $\tilde{\tau}_{*}$ can also be expressed in terms of the downstream variables with $\tilde{\tau}_{*}=\tilde{\tau} / M_{A}^{2}$. Hence the region $\tilde{\tau}>\tilde{\tau}_{*}$ corresponds to super-Alfvénic downstream flows, and $\tilde{\tau}<\bar{\tau}_{*}$ to sub-Alfvénic downstream flow speeds. Finally, the "Chapman-Jouguet" points are labeled I and II, and these points indicate where the downstream normal fluid velocity $u_{x}$ changes from supermagnetosonic to submagnetosonic. Thus the downstream states corresponding to a given upstream state are easily read off from the Hugoniot, and a more detailed survey of the parameter regime is presented in section 4 . 


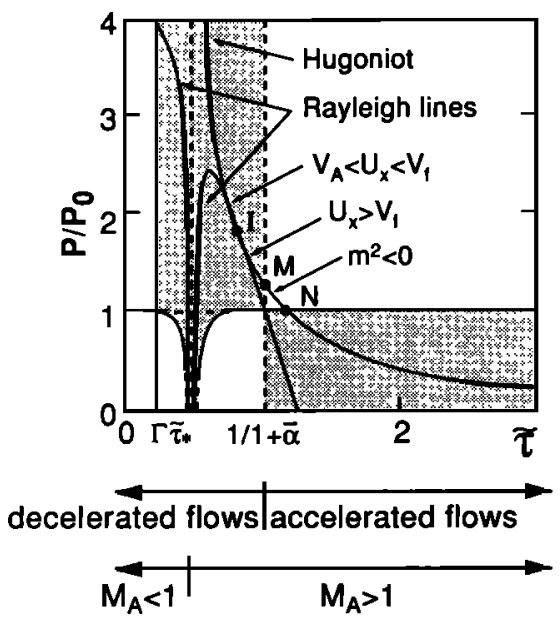

(a)

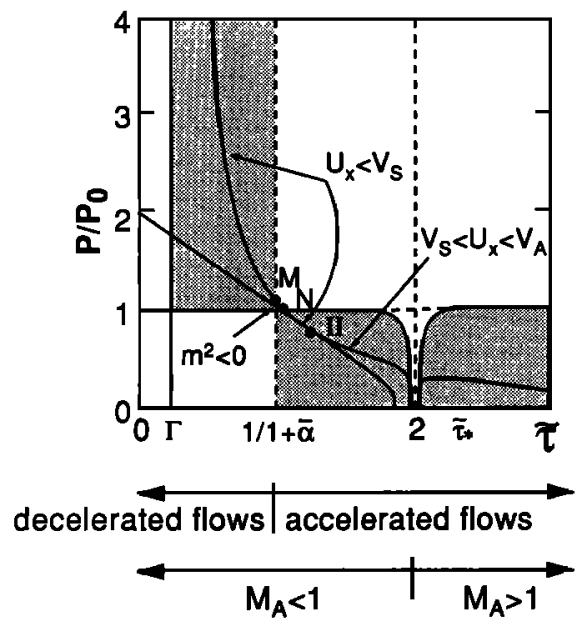

(b)

Fig. 6. Two illustrative examples of the Hugoniot and the Rayleigh curves, in which the different features are labeled and marked. (a) Incident flows satisfying $u_{x 0}>V_{f 0}$. (b) $V_{f 0}>u_{x 0}>$ $V_{s 0}$. The inequalities within the figures describe the downstream flow speeds corresponding to a particular segment of the Hugoniot. See text for further details. (Here $\theta_{0}=0^{\circ}, \gamma=5 / 3, \beta_{p 0}=1, \widetilde{V}_{y}=$ 4 , and $\bar{\alpha}=0.01$.)

\subsection{The Shock Polar Relation}

In concluding this section, we discuss briefly the massloading form of the shock polar relation and present an example which corresponds to that used in Figure 6. The mass-loading shock polar relation corresponding to equation (2) is derived by equating (58) and (51) and then using

$$
\bar{\tau}=\frac{1}{1+\bar{\alpha}} \frac{M_{A}^{2}}{M_{A 0}^{2}} .
$$

The final result is an extremely cumbersome fourth-order polynomial in $M_{A}^{2}$ which is not particularly revealing. Since the shock polar polynomial has to be solved numerically anyway, we may as well work with (51), (58), and (63) directly. We note in passing that the reason the mass-loading shock polar polynomial is an order greater than its classical counterpart is because the initial upstream state no longer lies on the mass-loading Hugoniot, i.e., a state cannot be connected to itself by a mass-loading front, or, equivalently,

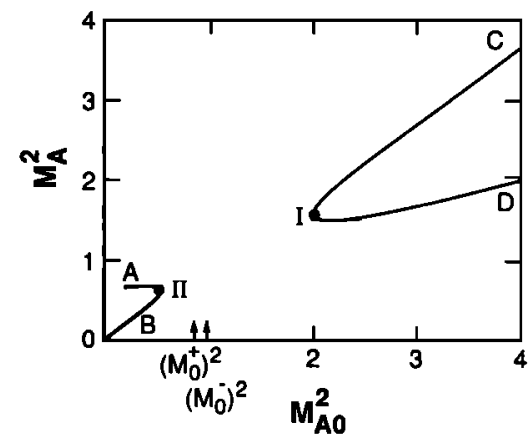

Fig. 7. Plot of the Alfvén Mach number shock polar relation corresponding to the parameters of Figure 6 . The squares of the normalized fast and slow magnetosonic speeds are labeled $\left(M_{0}^{+}\right)^{2}$ and $\left(M_{0}^{-}\right)^{2}$, respectively, and their values are indicated by the arrows in the figure. Only those real solutions to the R-H conditions for which $p>0$ are plotted.

that $M_{A}^{2}=M_{A 0}^{2}$ is not a solution to the mass-loading R-H conditions.

An example of a mass-loading shock polar relation is plotted in Figure 7, where the parameters of Figure 6 have been used. The Chapman-Jouguet points are again labeled I and II, and they clearly represent limiting points for which solutions to the mass-loading $\mathrm{R}-\mathrm{H}$ conditions exist. Furthermore, as discussed above, the turning points I and II identify the points at which the downstream normal flow speed is equal to one of the magnetosonic speeds; in this case, I corresponds to the fast and II to the slow magnetosonic speed. We have also marked the ratios of the fast and slow upstream magnetosonic speeds to the upstream Alfven speed with arrows, where we have introduced the notation

$$
\left(M_{0}^{-}\right)^{2}=V_{s 0}^{2} / V_{A 0}^{2}, \quad\left(M_{0}^{+}\right)^{2}=V_{f 0}^{2} / V_{A 0}^{2} .
$$

For parallel shocks we have $\left(M_{0}^{-}\right)^{2}=1$ and $\left(M_{0}^{+}\right)^{2}=$ $\gamma \beta_{p 0} / 2$. By comparing Figure $2 d$ and Figure 7 , it is evident how mass loading distorts the classical shock polar relation, and this distortion corresponds to the description given in section 3.1 .

To determine which sections of the shock polar illustrated in Figure 7 correspond to physically realizable mass-loading fronts, we utilize a geometric formulation of the entropy condition rather than requiring that the shock simply be compressive, as in section 2 . This is done in the following section. Figures 6 and 7 have been discussed, albeit briefly, in paper 2 .

\section{Classification of Mass-Loading MHD SHOCKS}

Lax's formulation of the entropy condition for hyperbolic systems of equations has been discussed in detail in paper 1 . For the sake of brevity then, we simply define the entropy condition here, make a few comments, and refer the interested reader to section 2 of paper 1 . Since the actual Lax inequalities are needed later in the analysis, a simplified derivation appropriate to stationary shocks is given in the appendix. We define the entropy condition as follows: A discontinuity satisfies the entropy condition if when it separates the characteristics of a family, the characteristics on each side can be traced back to the initial data.

A discontinuity is said to separate a family of character- 


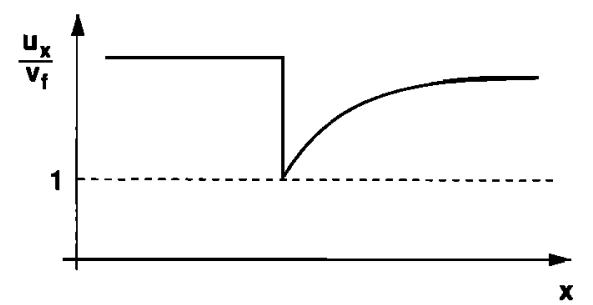

Fig. 8. Sketch of a mass-loading fast compound shock, illustrating the initial deceleration of the flow to the fast magnetosonic speed, before its recovery to the final downstream flow speed. The slow compound shock has qualitatively the same strucuture.

istics if through every point of the shock trajectory in the $(x$, $t$ ) plane, there exists a pair of characteristics which can both be traced either backward or forward in time. Motivation for these definitions can be found either in paper 1 or in Lax's monograph [Lax, 1973].

\subsection{Parallel Shocks}

This case has been discussed briefly in paper 2. For the sake of completeness, however, and also for the purposes of comparison, an abbreviated discussion of parallel massloading shocks is presented here. Two cases seem to be reasonably representative. The first case, $\beta_{p 0}=1$, is illustrated in Figures 6 and 7. We may identify the segment ID of Figure 7 with fast mode mass-loading shocks. The segment IC, however, corresponds to a quite different form of MHD shock, a compound shock (this is a consequence of the mass-loading MHD system being nonconvex; see paper 1). As shown in paper 2 , the compound mass-loading front consists of a shock followed by an isentropic centered rarefaction wave, the important point being that the initially shocked flow is decelerated only as far as the fast magnetosonic speed before being accelerated back to its final, although decelerated, downstream state. The structure of such a compound shock is sketched in Figure 8, and it was suggested in paper 2 that trains of such structures may have been observed at the Halley bow shock on the inbound Giotto encounter [Coates et al., 1987a, b; Neugebauer et $a l ., 1987 a$ ]. It should be noted that the inbound observations revealed that the bow shock was more perpendicular than parallel, and this is discussed more fully below and in the conclusions. A further point to note is that since we are not investigating the structure of the shock, we cannot on the basis of our MHD formulation estimate the size of the compound shock illustrated in Figure 8. In a sense, we have already prescribed the overall shock dimensions by our prescription of $\alpha$.

The segments AII and BII correspond to accelerated (expansion) flows; in the case of AII the transition is subsonic-super(slow)magnetosonic, whereas for BII the transition is simply subsonic-subsonic. It was proved in paper 2 that the R-H solutions described by AII were inadmissible but that the region parameterized by BII was indeterminate (i.e., it could not be decided on the basis of the entropy condition alone whether these regions represented sensible solutions to the mass-loading R-H conditions). To determine whether the solutions corresponding to BII are really allowed, one needs to consider the full shock structure problem including the appropriate dissipative mechanisms. Perhaps the most startling difference between these shock
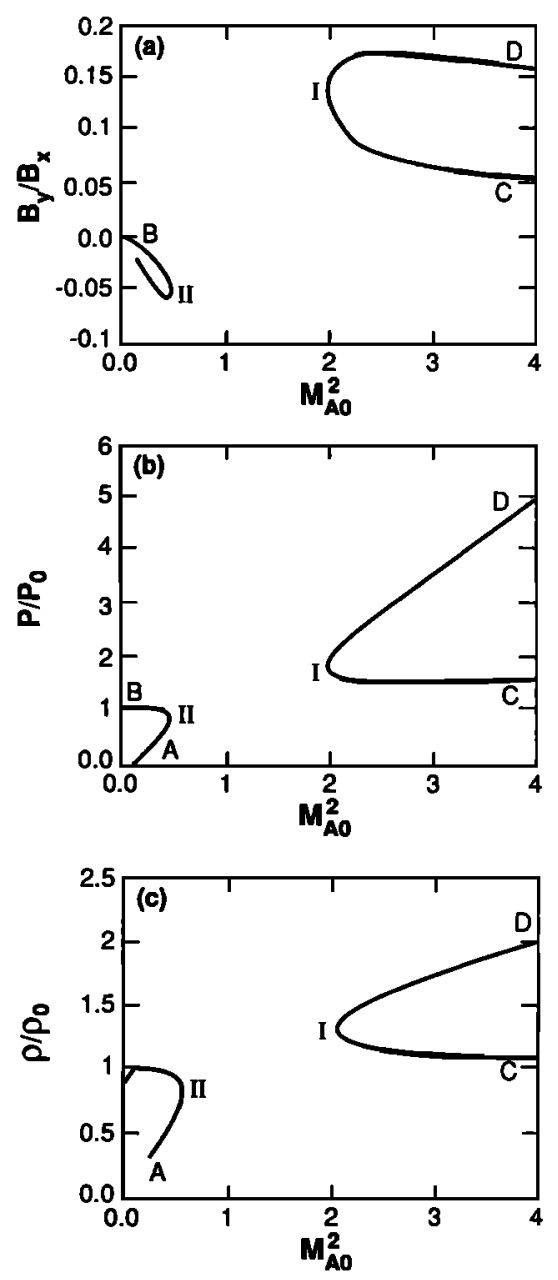

Fig. 9. Plots of (a) the downstream tangential magnetic field, $(b)$ the downstream thermal gas pressure, and $(c)$ downstream density as functions of the square of the incident Alfven Mach number of the flow for the parallel shock of Figure 6. Of particular importance is the nonzero tangential magnetic field component, even though the shock was initially parallel.

polar plots and those of section 2 is the very large parameter regime which does not admit solutions to the mass-loading R-H conditions. In other words, mass loading severely curtails the possibility of a flow possessing simple structures which take the supersonic incoming flow to a subsonic downstream state. Instead, we might expect the overall shock structure to consist of a complicated combination of fast or slow mode shocks, compound shocks, and slip lines.

The downstream tangential magnetic field, pressure, and density are plotted as functions of the square of the incident flow Alfvénic Mach number $M_{A 0}^{2}$ in Figure 9. Two points are worth noting. The first is that although the shocks are parallel, the downstream tangential magnetic field is always "switched on" (see equation (52) with $\theta_{0}=0$ ). However, because $M_{A 0}$ can never approach 1, extremely strong rotations of the magnetic field cannot occur, although more modest rotations of up to about $10^{\circ}$ are possible. This appears to be insufficient to account fully for the observed downstream magnetic field described by Neubauer et al. [1990]. Nevertheless, a definite answer to the possibility of very strong downstream magnetic field rotations at massloading shocks can be achieved only by considering the full shock structure problem. This is because the field will begin 


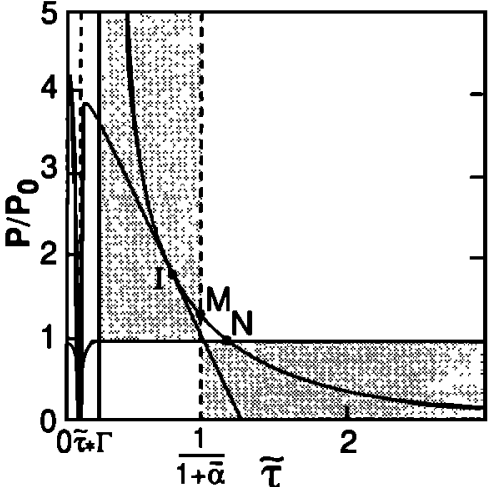

(a)

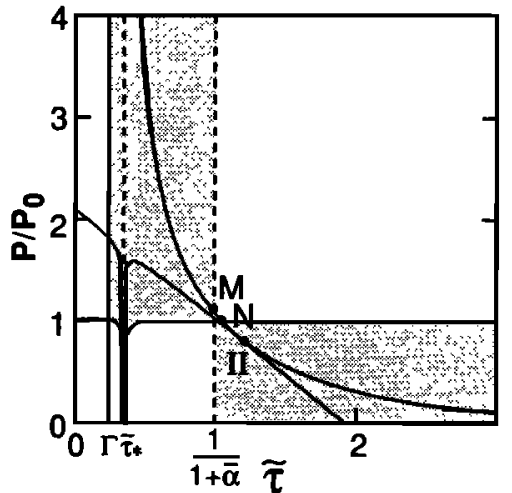

(b)

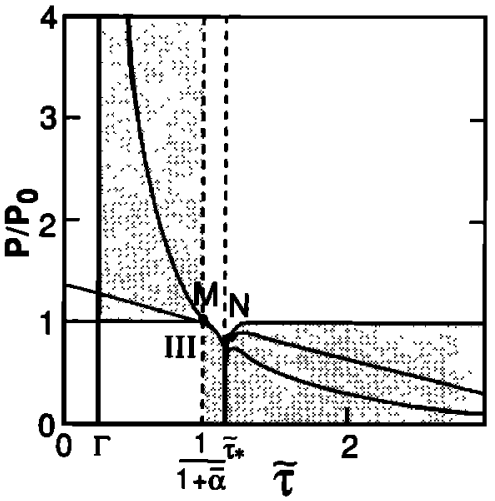

(c)

Fig. 10. Plots of the Hugoniot and Rayleigh curves for a large plasma beta $\left(\beta_{p 0}=5, \theta_{0}=0^{\circ}, \gamma=5 / 3, \bar{V}_{y}=4\right.$, $\bar{\alpha}=0.01$ ), but otherwise with the same parameters as used in obtaining Figure 6. Observe now the presence of three "Chapman-Jouguet" points. The notation is the same as in Figure 6.

to "twist" or rotate in the smooth but decelerating (foreshock) part of the flow. In addition, the flow in the foreshock region is also homogeneous, and further complications may arise through the possibility that the pressure tensor may be somewhat anisotropic. The second point concerns those solutions of the R-H conditions that admit solutions with negative gas pressure but otherwise sensible fluid parameters. In this case the downstream pressure is provided fully by the strongly rotated magnetic field, but as we have demonstrated, these solutions are patently unphysical.

Consider now the case with $\beta_{p 0}=5$, which may be more consistent with the plasma parameters measured at Halley. The Hugoniot and Rayleigh curves are plotted in Figure 10, and we see that three distinct points of tangency (labeled I, II, and III) exist, each of which corresponds to a different upstream state. This is illustrated nicely in the shock polar plot Figure 11. Again, it is immediately noticeable on comparing Figure $11 b$ to its classical counterpart in section 2 that large regions of upstream parameter space no longer admit real solutions to the mass-loading R-H conditions. Owing to the complexity of Figures 10 and 11, we show in detail the nature of the possible mass-loading fronts directly in Figure 11a. As in the $\beta_{p 0}=1$ case, IE corresponds to fast mode mass-loading shocks, and ID to compound super(fast)magnetosonic-super(fast)magnetosonic shocks. However, apart from segments CII and AIII, which are both indeterminate, all the remaining segments of Figure $11 b$ represent inadmissible R-H solutions. Plots of the downstream tangential magnetic field, pressure, and density as functions of $M_{A 0}^{2}$ are exhibited in Figure 12.

Before concluding this subsection, observe that for parallel shocks, stable slow mode mass-loading fronts do not exist.

\subsection{Oblique Shocks}

In this subsection we prove in some detail the existence and nonexistence of various kinds of oblique mass-loading shocks. Our analysis is guided and illuminated by the simultaneous use of the shock polar relation and the corresponding Hugoniot. In this manner we can easily compare the more complicated mass-loading MHD results with the classical MHD results of section 2 .

4.2.1. $\theta_{0}=5^{\circ}$. Consider first the quasi-parallel case $\theta_{0}=$ $5^{\circ}\left(\bar{\alpha}=0.01, \bar{V}_{y}=4, \beta_{p 0}=1\right.$, and $\left.\gamma=5 / 3\right)$. The shock polar relation is illustrated in Figure $13 a$, and Figures $13 b, 13 c$, and $13 d$ show the downstream tangential magnetic field, pressure, and density, respectively, as functions of the square of the incident Alfvén Mach number. Evidently, no great differences exist between quasi-parallel and parallel mass-loading fronts, and the results of section 4.1 carry through essentially unchanged for this case.
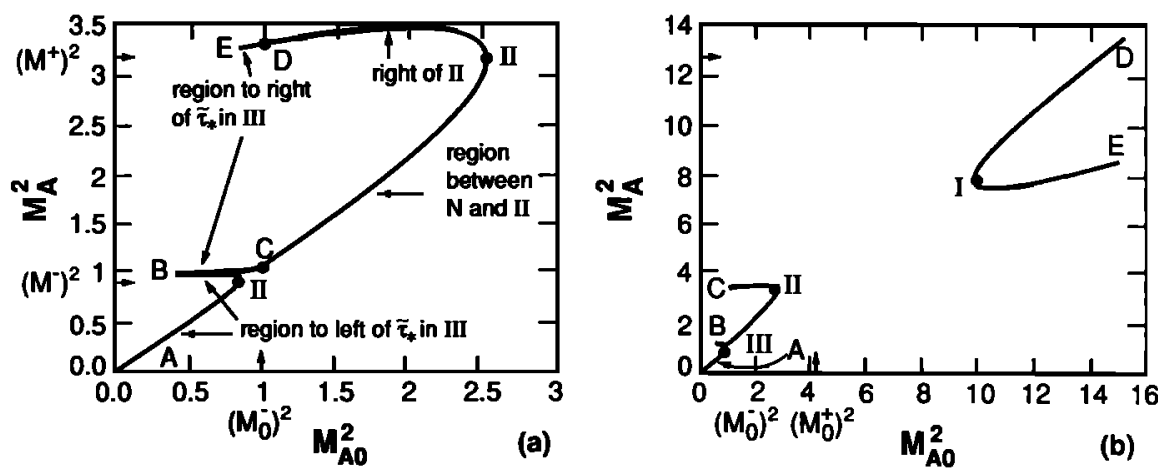

Fig. 11. The shock polar curves corresponding to Figure 10. (a) Detail of the "slow" section of the shock polar is displayed, together with the correspondence to the various sections to the Hugoniot plots of Figure 10. Note that II and III correspond to the downstream fast and slow magnetosonic speeds and that only those sections of the shock polar corresponding to $p>0$ are plotted. (b) The complete shock polar curve. 

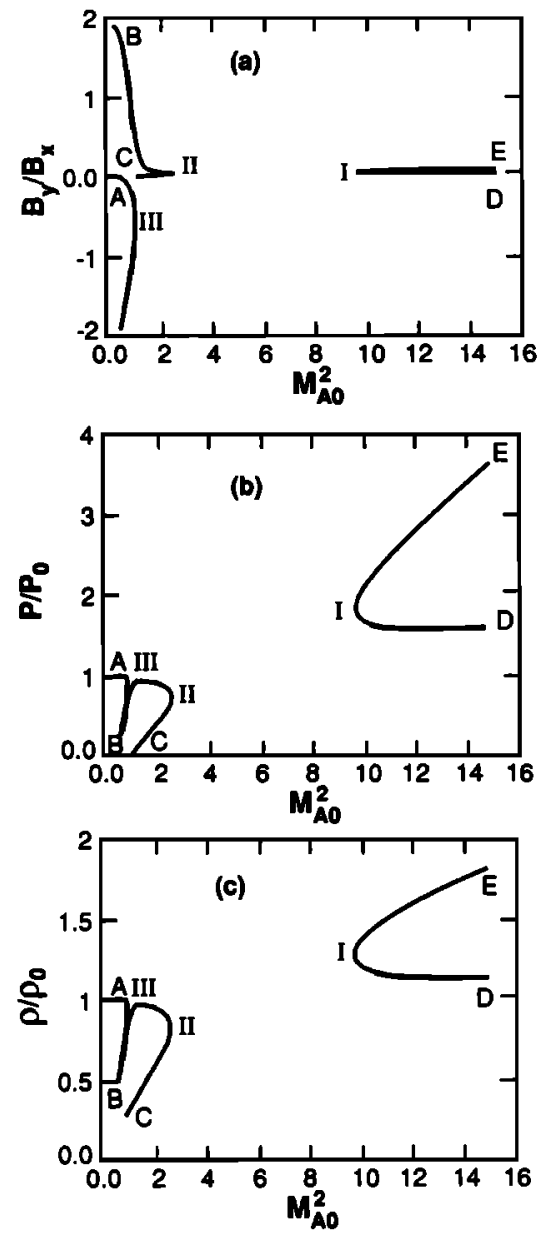

Fig. 12. Plots of $(a)$ the downstream tangential magnetic field, (b) the downstream thermal gas pressure, and (c) downstream density as functions of the square of the Alfven Mach number of the incident flow for the large plasma beta parallel shock of Figure 10.

4.2.2. $\theta_{0}=30^{\circ}$. On increasing the obliquity of the incident magnetic field, we find a third class of solutions to the R-H conditions. The new solutions correspond to the "figure-eight" topological feature exhibited in the shock polar plot of Figure $14 a$ (where the parameters $\theta_{0}=30^{\circ}, \bar{\alpha}=$ $0.01, \bar{V}_{y}=4, \beta_{p 0}=1$, and $\gamma=5 / 3$ have been used). On comparing Figure $14 a$ to its classical counterpart (Figure $2 b$ ), it is evident that the $M_{A}^{2}=M_{A 0}^{2}$ graph and the cubic polynomial (2) have been "fused" by the addition of mass to the system. The topological distortion of the classical MHD shock polar relation is clearly consistent with the description of section 3. Note that in Figure $14 a,\left(M_{+}^{0}\right)^{2}=V_{f 0}^{2} / V_{A 0}^{2} \simeq$ 1.8 and $\left(M_{-}^{0}\right)^{2}=V_{s 0}^{2} / V_{A 0}^{2} \simeq 0.6$, where, using (25), we have

$$
\begin{aligned}
\left(M_{ \pm}^{2}\right)^{2} & =\frac{1}{2}\left(\frac{\gamma}{2} \beta_{p 0}+1\right) \sec ^{2} \theta_{0} \\
\pm & \frac{1}{2}\left[\left(\frac{\gamma}{2} \beta_{p 0}+1\right)^{2} \sec ^{4} \theta_{0}-2 \gamma \beta_{p 0} \sec ^{2} \theta_{0}\right]^{1 / 2}
\end{aligned}
$$

The points labeled I-VI correspond to six possible touching conditions for the Hugoniot and the Rayleigh curves. At each point, the downstream normal flow velocity $u_{x}$ is equal to either the fast or the slow magnetosonic speed. The Hugoniots corresponding to each tangent point are shown in
Figure 15. As before, the segment of the Hugoniot between points $\mathrm{M}$ and $\mathrm{N}$ corresponds to those $\mathrm{R}-\mathrm{H}$ solutions for which $m^{2}<0$. We consider each of the different Hugoniots in turn.

Consider a constant state $S$ which corresponds to a point $\left(\bar{\tau}_{1}, p_{1}\right)$ lying above point $\mathbf{M}$ on the mass-loading Hugoniot of Figure 15a. All possible states of interest correspond to decelerating mass-loading fronts (i.e., because all the $\tilde{\tau}$ of interest satisfy $\tilde{\tau}<1 /[1+\bar{\alpha}]$ ), but since $\bar{\tau}>\bar{\tau}_{*}$, the downstream flow speeds are all super-Alfvénic (we consider the section of the Hugoniot below $\mathrm{N}$ later). Depending on the precise location of $S$, we need to consider three cases.

Strong compression: $S$ lies in the strong compression branch $p_{1}>p_{\mathrm{I}}$. From the shock polar plot (Figure 14a) it is evident that $u_{x 0}>V_{f 0}$. Since I corresponds to the downstream state at which $u_{x}=V_{f}$, where $V_{f}$ is the fast magnetosonic speed, the downstream normal flow speed corresponding to the state $S$ satisfies the inequality $V_{A 1}<$ $u_{x 1}<V_{f 1}$. Accordingly, the characteristics (24) assume the geometric configuration depicted in Figure 16 for a stationary left-facing shock. (There is no additional difficulty in considering nonstationary shocks.) The mass-loading front separates the $C_{f}^{-}$family of characteristics, whereas the other families simply cross the front (we plot only the $C^{-}$ characteristics to avoid cluttering the figure, since the shock is left-facing). Clearly, the $C_{f}^{-}$characteristics can also be traced back to the initial data; hence the strong compression solutions associated with the fast magnetosonic characteristics satisfy the entropy condition. Thus the downstream state is determined uniquely from the upstream state. We can therefore identify the region I-B of the shock polar plot of Figure 14 as the mass-loading analogue of classical fast mode MHD shocks.

$S=I:$ In this case we have $p_{1}=p_{1}$, and the flow velocity upstream of the mass-loading front satisfies $u_{x 0}>$ $V_{f 0}$ whereas downstream $u_{x 1}=V_{f 1}$. Thus one of the downstream characteristics, in this case $C_{f}^{-}$, when viewed from downstream, coincides with the mass-loading front, illustrated in Figure 17. Clearly, the condition $u_{x}=V_{f}$ downstream of the front enables us to determine the downstream rate from the upstream without further assumption.

Weak compression: As described in papers 1 and 2 , the region of the Hugoniot satisfying $p_{\mathrm{I}}>p_{1}>p_{\mathrm{M}}$ is particularly interesting. Again, the upstream normal flow velocity satisfies $u_{x 0}>V_{f 0}$, but as can be seen from the touching condition, the gas flow downstream of the front also satisfies $u_{x 1}>V_{f 1}$. However, beause $\bar{\tau}<1 /(1+\bar{\alpha})$, the incoming flow is decelerated on passage through the mass-loading front. As illustrated in Figure 14c, we always have $p_{1}>p_{0}$. Each family of characteristics crosses the front (Figure 18), and as discussed in paper 1 , this implies that the massloading MHD system of equations is nonconvex. Under these conditions, $S$ and $\left(\tau_{0}, p_{0}\right)$ cannot be connected by a single wave front. It was shown by Oleinik [1959] that to exclude discontinuities that move faster than the characteristic speeds on either side of the front, it is necessary to construct a compound wave connecting upstream and downstream states. This is accomplished by connecting the upstream state to the $S=I$ front and then following by an isentropic centered rarefaction wave to reach the downstream state. Such a compound wave is possible because the $S=I$ front moves at the downstream fast magnetosonic speed. If we use such a compound wave to connect the 


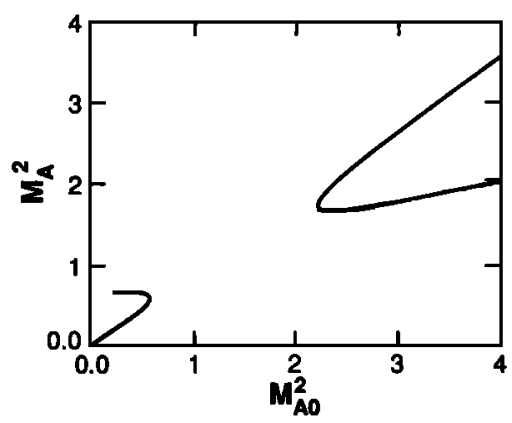

(a)

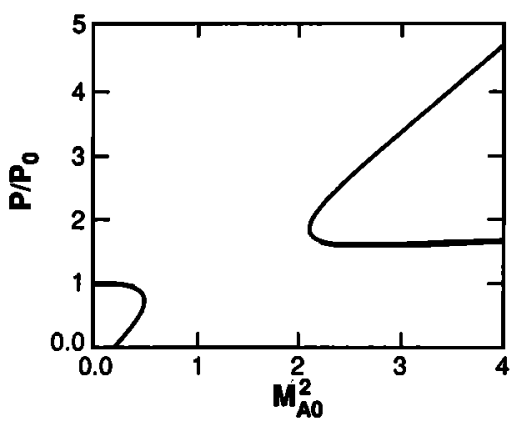

(c)

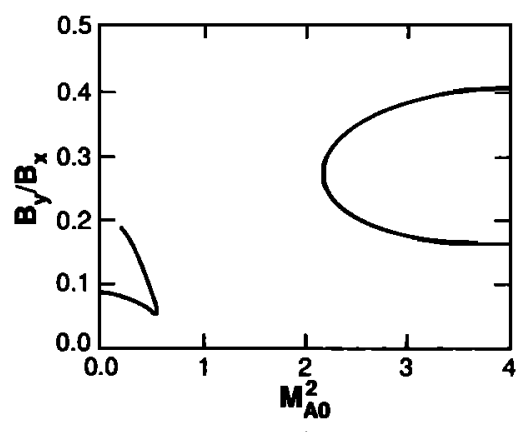

(b)

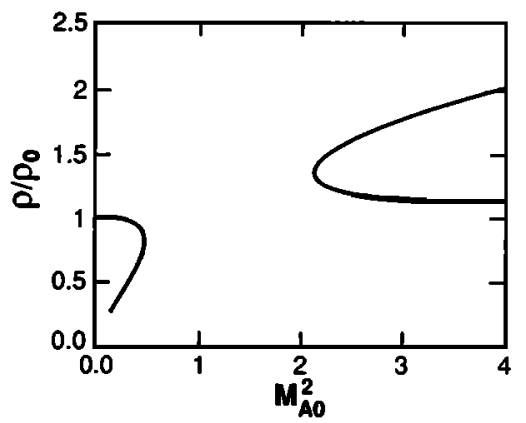

(d)

Fig. 13. (a) The Alfvenic Mach number shock polar curve together with plots of (b) the downstream tangential magnetic field, $(c)$ the downstream thermal gas pressure, and $(d)$ downstream density as functions of the square of the Alfven Mach number of the incident flow for the quasi-parallel case $\theta_{0}=5^{\circ}\left(\beta_{p 0}=1, \gamma=5 / 3, \tilde{V}_{y}=4\right.$, and $\left.\bar{\alpha}=0.01\right)$. Note the similarity between this example and the parallel shock case above.

states, then the downstream state is determined completely by the upstream state. Had we not excluded weak compression discontinuities, then the solution to the initial-value problem would not be unique. Thus the section IA on the shock polar diagram in Figure $14 a$ is associated with "fast compound" mass-loading MHD shocks, and these shocks have no classical analogue. The possibility that fast compound shocks were observed on the inbound leg of the Giotto-Halley encounter is discussed in section 5 .

Consider now the region D-IV-II-D of the shock polar

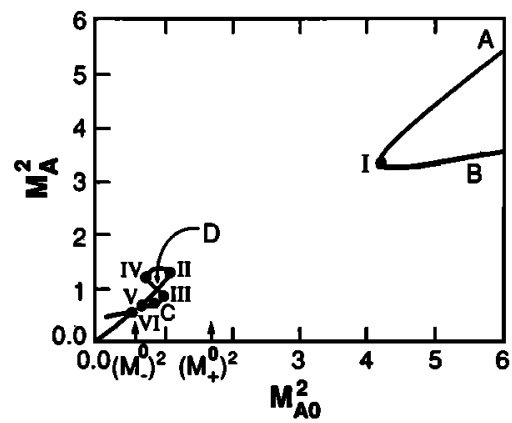

(a)

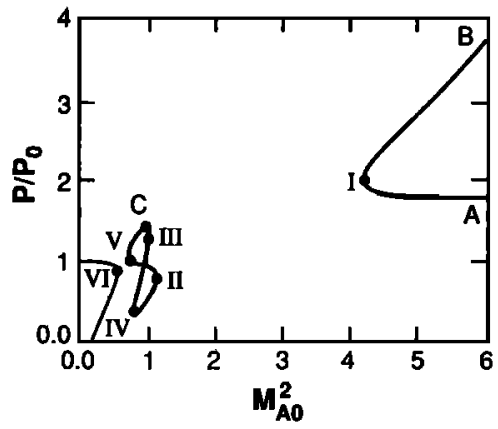

(c)

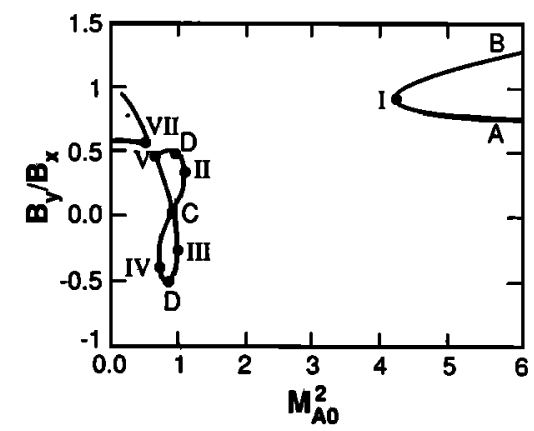

(b)

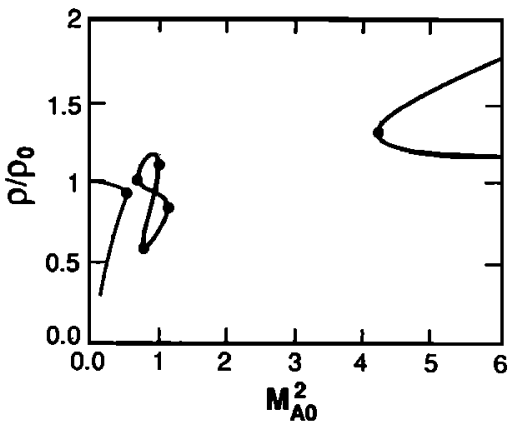

(d)

Fig. 14. As in Figure 13, except now $\theta_{0}=30^{\circ}$. Observe, in particular, the presence of the new "figure eight" topology. 


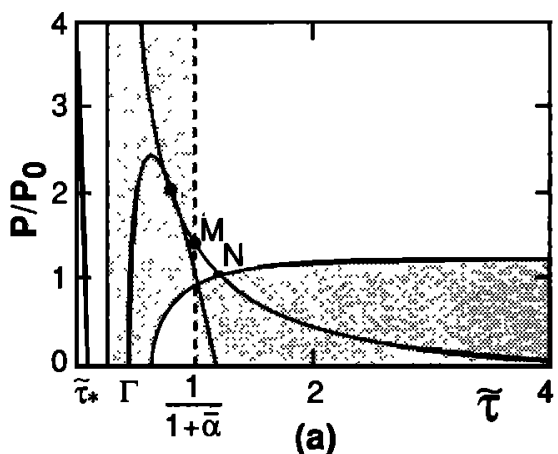

(a)
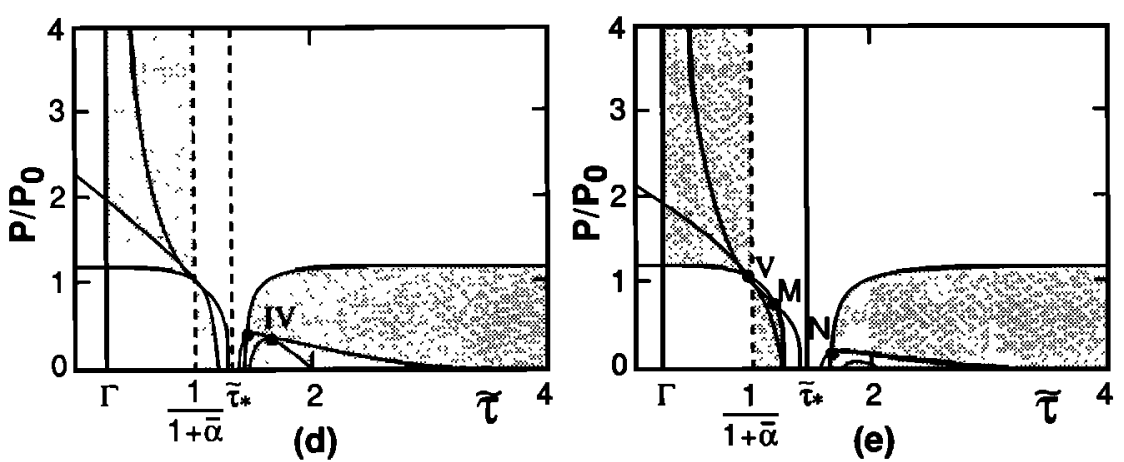

(e)
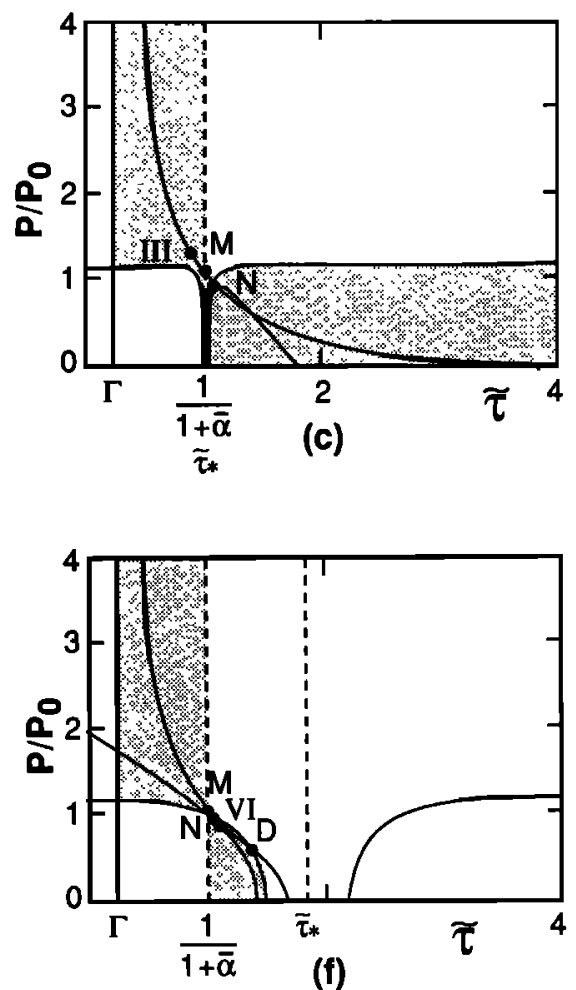

Fig. 15. The Hugoniot and Rayleigh curves appropriate to each of the mass-loading "Chapman-Jouguet" points for the $\theta_{0}=30^{\circ}$ example. In conjunction with the shock polar curve of Figure 14, these figures are sufficient to provide a complete characterization of these mass-loading fronts. See text for details.

curve (Figure 14a). The Hugoniots appropriate to this section of the figure eight are depicted in Figures $15 b$ and $15 d$. For these cases we have $\bar{\tau}>\bar{\tau}_{*}$, from which we infer immediately that mass-loading fronts in this parameter regime must be expansive.

Having illustrated the general arguments for determining the existence and nonexistence of mass-loading fronts in some detail, we can afford to be brief. The cases that need to be considered are

Case 1

$$
\begin{aligned}
& p<p_{\text {II }} \\
& p<p_{\text {IV }}
\end{aligned}
$$

in which case $u_{x}>V_{f}$;

Case 2

$$
\begin{aligned}
& p>p_{1 \mathrm{l}} \\
& p>p_{\mathrm{IV}}
\end{aligned}
$$

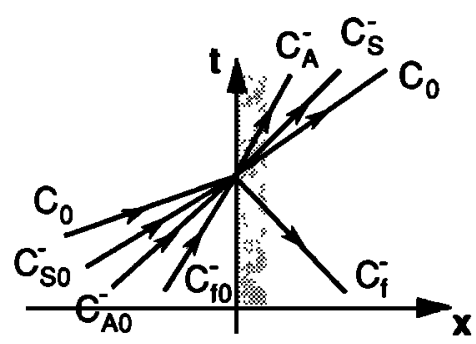

Fig. 16. The characteristics for the strong compression regime corresponding to Figure $15 a$. The shaded region denotes the stationary left-facing front. To avoid crowding the figure, we plot only the negative characteristics. for which $V_{A}<u_{x}<V_{f}$; and

Case 3

$$
p=p_{\text {II }}, p_{\text {IV }}
$$

so that $u_{x}=V_{f}$. The upstream normal flow speeds can satisfy either $u_{x 0}>V_{A 0}>V_{s 0}$ or $V_{A 0}>u_{x 0}>V_{s 0}$ (Figure 14).

For case 1 we can have either $V_{s 0}<u_{x 0}<V_{A 0}, u_{x}>V_{f}$ or $V_{f 0}>u_{x 0}>V_{A 0}, u_{x}>V_{f}$, the former inequality corresponding to the space-time configuration illustrated in Figure $19 a$ and the latter to that shown in Figure $19 b$. For both possible cases the $C_{f}^{-}$characteristic is separated by the stationary left-facing shock, but these characteristics, although separated, can never be traced back to the initial data. Hence the segment II-IV represents inadmissible solutions to the mass-loading $\mathrm{R}-\mathrm{H}$ conditions.

In case 2 above, we have either $V_{s 0}<u_{x 0}<V_{A 0}, V_{A}<$ $u_{x}<V_{f}$ or $V_{A 0}<u_{x 0}<V_{f 0}, V_{A}<u_{x}<V_{f}$, with the space-time diagrams illustrated in Figures $20 a$ and $20 b$, respectively. Since the family of $C_{A}^{-}$characteristics in

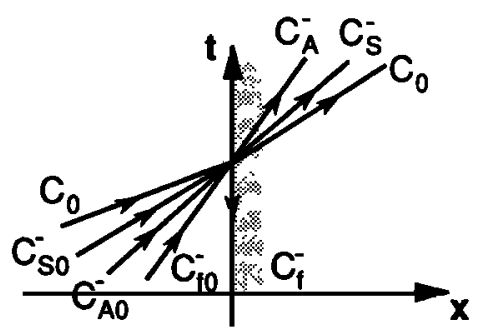

Fig. 17. As in Figure 16, except now for the case $S=\mathrm{I}$. 


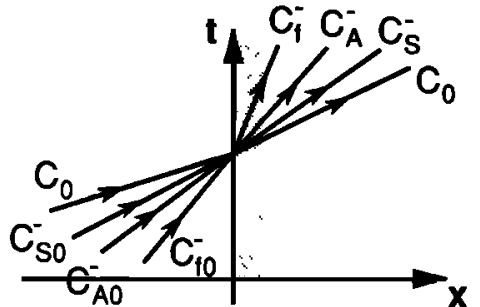

Fig. 18. As in Figure 16, except now for the weak compression regime.

Figure $20 a$ cannot be traced backward in time, no massloading shocks can exist in this parameter regime. The families of characteristics illustrated in Figure $20 \mathrm{~b}$ are a little different from those discussed already. At first sight, one might conclude that this case is similar to that illustrated in Figure 18. However, because the upstream characteristic $C_{f 0}^{-}$can never be traced backward in time, a compound wave can never be constructed sensibly to connect the upstream to the downstream state. Thus the segment IV-D-II is inadmissible for mass-loading fronts. Consequently, the entire region D-IV-II-D does not represent legitimate massloading solutions to the conservation laws.

The lower half of the figure eight, i.e., D-V-C-III-D, is more interesting and related to the slow mode and intermediate shock section of the classical MHD shock polar curve. The two Hugoniots appropriate to this section of the massloading shock polar curve are depicted in Figures $15 \mathrm{c}$ and $15 e$. Points III and V of Figure 14 therefore correspond to those states for which the downstream fluid velocity is equal to the slow magnetosonic speed. An interesting feature of the lower section of the figure eight is point $C$. Inspection of Figures $14 b$ and $14 c$ indicates that $C$ corresponds to a mass-loading front for which the downstream tangential magnetic field is switched off, i.e., $B_{y}=0$, and consequently the local downstream gas pressure is maximized for these non-fast mode mass-loading fronts. Obviously, C corresponds to the switch-off shock of classical MHD except that now $M_{A 0} \neq 1$.

Let us consider the Hugoniot segment for which $p>p_{\text {III }}$. In this case we have $V_{s 0}<u_{x 0}<V_{A 0}, u_{x}<V_{s}$ (Figures 14 and $15 c$ ), and so the space-time diagram for a stationary left-facing shock is as sketched in Figure $21 a$. Clearly, the $C_{s}^{-}$family of characteristics satisfy the geometric entropy condition, indicating that this family of solutions to the mass-loading $\mathrm{R}-\mathrm{H}$ conditions represent valid shock waves. These shocks are exactly analogous to the slow mode shocks of nonreacting MHD shock theory. At the point $p=p_{\text {III }}$, we have $V_{s 0}<u_{x 0}<V_{A 0}, u_{x}=V_{s}$, and the flow is decelerated. This time, the downstream characteristic $C_{s}{ }^{-}$is rotated until it lies parallel to the mass-loading front (Figure $21 b$ ). As discussed above, this too is a perfectly valid space-time configuration in terms of the geometric entropy condition, and so the downstream state can be determined from the upstream without further assumption. Consider finally the regime $p_{\mathrm{M}}<p<p_{\text {III }}$ on the Hugoniot of Figure $15 c$. This corresponds to the region II-D of the shock polar plot (Figure 14), and we have $V_{s 0}<u_{x 0}<V_{A 0}, u_{x}<V_{A}$, although $u_{x}<u_{x 0}$. In this case the $C_{s}^{-}$characteristics form a nonconvex family (Figure $21 c$ ). As described already, it is necessary to use a compound wave to connect upstream and downstream states, but this time we use the slow magnetosonic front (Figure $21 b$ ) followed by an isentropic centered rarefaction wave. Thus, in addition to finding fast magnetosonic compound waves, we have also established the existence of slow magnetosonic compound waves, both of which form a completely new class of shocks and neither of which has any counterpart in classical MHD shock theory.

An analysis precisely analogous to that used in discussing the "Chapman-Jouguet" point III holds for the distinguished point $\mathrm{V}$. Thus the region $\mathrm{V}-\mathrm{C}$ of the shock polar plotted in Figure 14 describes slow mode mass-loading shocks in which the downstream tangential magnetic field is decreased until it is switched off completely at C. On the segment C-III the mass-loading shock possesses properties of both classical slow mode and intermediate MHD shocks. On the one hand, the shock is sub-Alfvénic, yet the downstream magnetic field is rotated into an opposite orientation (Figure $14 b$ ). Finally, the segment V-D-III represents the slow mode compound shock discussed above.

It is easily established that the remaining sections of the shock polar curve associated with the Chapman-Jouguet point VI (Figures 14 and 15f) are either indeterminate or inadmissible as far as solutions to the mass-loading $\mathrm{R}-\mathrm{H}$ conditions are concerned. This then completes the analysis of mass-loading shocks which possess shock polar topologies of the kind exhibited in Figure $14 a$.

4.2.3. $\theta_{0}=45^{\circ}$. If the obliquity of the incident magnetic field is increased to about $45^{\circ}$, then the topology of the shock polar curves undergoes a further transition. This new structure is shown in Figure 22, where it is seen that the figure eight has now merged with (and part has split from) what was formerly the lower hyperbolic section of the shock polar curve. Furthermore, there now exist only three ChapmanJouguet points at which the downstream flow speed matches

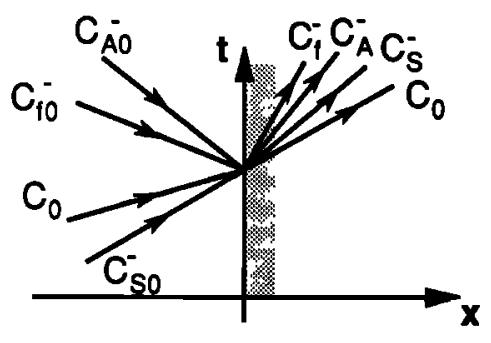

(a)

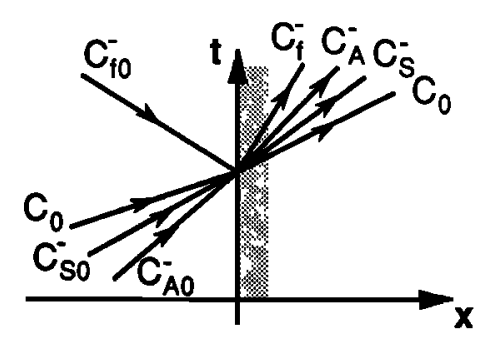

(b)

Fig. 19. The space-time diagram for when the downstream gas pressure satisfies $p<p_{\mathrm{II}}, p<p_{\mathrm{IV}}$; see Figures $15 b$ and $15 d$. See text for details. 


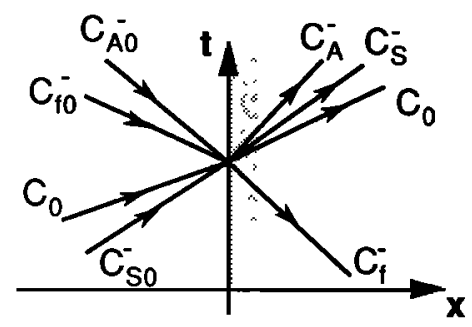

(a)

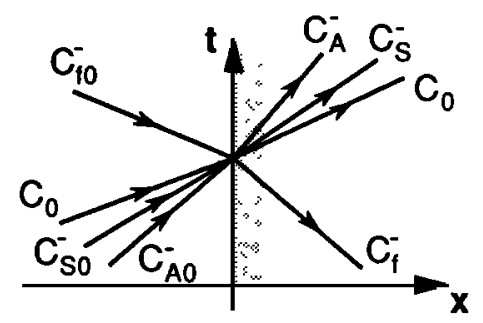

(b)

Fig. 20. As for Figure 19, except now $p>p_{\mathrm{II}}, p>p_{\mathrm{IV}}$.

the fast or slow magnetosonic speed. The associated Hugoniot plots are presented in Figure 23. The upper hyperbolic section of the shock polar curve A-I-B is very similar to that of the $5^{\circ}$ and $30^{\circ}$ cases, with I-B corresponding to the usual fast mode mass-loading shocks and I-A to the fast mode compound shocks.

The most interesting aspect of the $\theta_{0}=45^{\circ}$ example is the segment D-III-E-F of the shock polar curve (Figure 22). We need to use the Hugoniot plotted in Figure 23c. The three cases that we consider are

Case 1

$$
V_{f 0}>u_{x 0}>V_{A 0}>V_{s 0} \text { and } u_{x}<V_{s}<V_{A},
$$

Case 2

$$
V_{f 0}>u_{x 0}>V_{A 0}>V_{s 0} \text { and } u_{x}=V_{s}
$$

Case 3

$$
V_{f 0}>u_{x 0}>V_{A 0}>V_{s 0} \text { and } V_{s}<u_{x}<V_{A} .
$$

Note that point $\mathrm{F}$ of Figure 22 corresponds to the point at which the upstream normal flow speed matches the upstream flow magnetosonic speed, III is the point at which the downstream normal flow velocity matches the downstream slow magnetosonic speed, and $\mathrm{E}$ (for which $u_{x}<V_{s}$ ) locates the mass-loading switch-off front. An important difference between this and the $\theta_{0}=30^{\circ}$ case is the existence here of solutions to the mass-loading $\mathrm{R}-\mathrm{H}$ conditions for which $u_{x 0}>V_{A 0}$. The implications of having a class of solutions with $u_{x 0}>V_{A 0}$ are illustrated in Figures $24 a$ and $24 b$; i.e., there exist two distinct families of characteristics, $C_{A}^{-}$and $C_{s}^{-}$, for which the geometrical entropy condition appears to be adequately satisfied. However, since the idea behind the geometrical entropy condition was to ensure that the shock problem possessed a unique solution, it is not clear at all that cases 1 and 2 therefore correspond to valid solutions to the mass-loading R-H conditions. On the other hand, the spacetime diagram appropriate to case 3 above (Figure 24c) indicates that the family of $C_{\bar{A}}^{-}$characteristics are separated by the stationary left-facing shock and can be traced back uniquely to the initial data. These mass-loading fronts therefore exist and are in fact analogous to the intermediate shocks of classical MHD since the downstream magnetic field is rotated well into the opposite orientation (the downstream obliquity can be as much as $\sim 35^{\circ}$ downstream of the shock; see Figure 22b).

To understand what Figures $24 a$ and $24 b$ imply in terms of admissible solutions to the R-H conditions, it is necessary to go back to the original definition of Lax's entropy condition that underlies our simple geometric interpretation. Denote by $\left(\mathbf{u}_{l}, \mathbf{u}_{r} ; s\right)$ discontinuous solutions to the R-H conditions, where $u_{l}$ represents the left or upstream state and $u_{r}$ the right or downstream state. As before, $s$ denotes the propagation velocity of the mass-loading front. In the case of stationary shocks, $s=0$. Let $\lambda_{k}, 1 \leq k \leq n$, denote the eigenvalues of the hyperbolic system of equations of interest. Then a discontinuity $\left(\mathbf{u}_{l}, \mathbf{u}_{r}\right)$ is an admissible solution to the $\mathrm{R}-\mathrm{H}$ conditions if, for some index $k, 1 \leq k \leq n$, the following inequalities hold [Lax, 1973]:

$$
\begin{gathered}
\lambda_{k}\left(\mathbf{u}_{r}\right)<0<\lambda_{k}\left(\mathbf{u}_{l}\right) ; \\
\lambda_{k-1}\left(\mathbf{u}_{l}\right)<0<\lambda_{k+1}\left(\mathbf{u}_{r}\right),
\end{gathered}
$$

A brief discussion and simplified derivation of the inequalities (66) is to be found in the appendix. The inequalities (66) demonstrate that for only one index $k$ is the shock speed intermediate to the characteristic speeds $\lambda_{k}$ on either side of the shock. In fact, (66) is a form of stability condition since

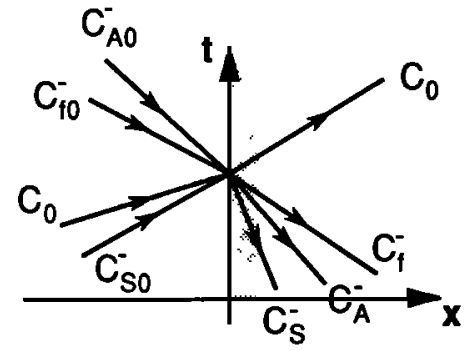

(a)

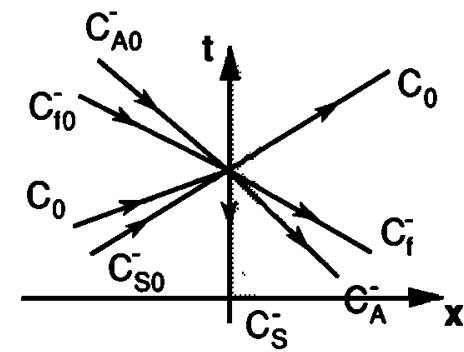

(b)

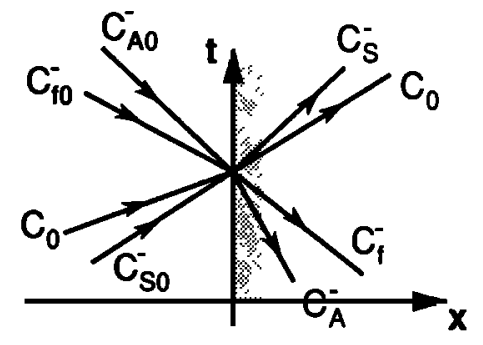

(c)

Fig. 21. The space-time diagram corresponding to Figure $15 c$ : (a) $p>p_{\mathrm{III}},(b) p=p_{\mathrm{III}}$, and (c) $p_{\mathrm{M}}<p<p_{\mathrm{III}}$ These figures should be interpreted in conjunction with the shock polar relation illustrated in Figure 14. 


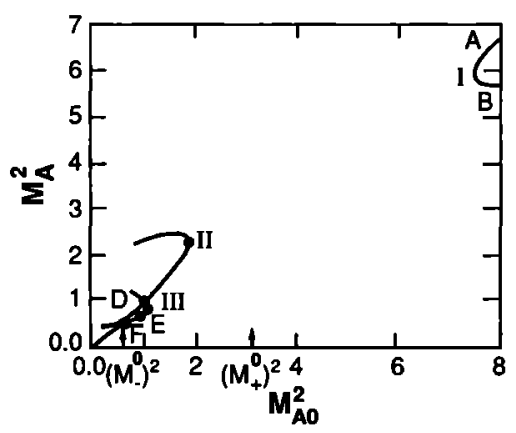

(a)

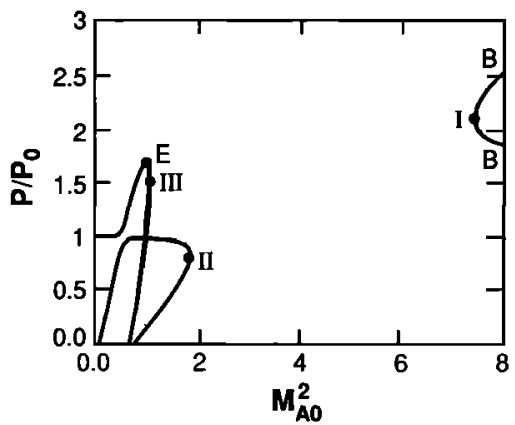

(c)

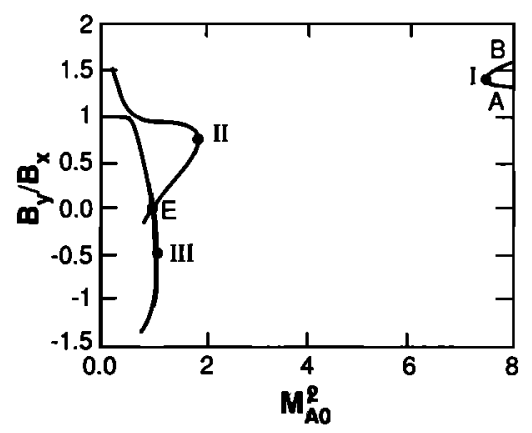

(b)

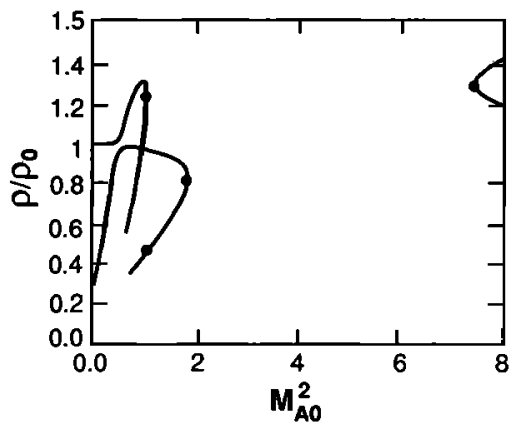

(d)

Fig. 22. (a) The shock polar curve, together with plots of $(b)$ the downstream tangential magnetic field, (c) the downstream thermal gas pressure, and $(d)$ downstream density as functions of $M_{A 0}^{2}$ for $\theta_{0}=45^{\circ}\left(\beta_{p 0}=1, \gamma=5 / 3\right.$, $\widetilde{V}_{y}=4$, and $\bar{\alpha}=0.01$ ). Note the existence of only three "Chapman-Jouguet" points and the merging of the figure eight with what was formerly the lower hyperbolic section of the shock polar curve.

it persists under small perturbations [e.g., Liberman and Velikovich, 1986].

Consider the left state

$\mathbf{u}_{l}$ :

$$
V_{s 0}<V_{A 0}<u_{x 0}<V_{f 0},
$$

and the right state

$\mathbf{u}_{\mathbf{r}}$ :

$$
u_{x}<V_{s}<V_{A}<V_{f}
$$

Then the eigenvalues (24) (i.e., $\lambda_{0}=u_{x}, \lambda_{s, f, A}^{ \pm}=u_{x} \pm$ $V_{s, f, A}$ ) to the left of the stationary shock are ordered as follows:

$$
\lambda_{f 0}^{-}<0<\lambda_{A 0}^{-}<\lambda_{s 0}^{-}<\lambda_{0}\left(\mathbf{u}_{l}\right)<\lambda_{s 0}^{+}<\lambda_{A 0}^{+}<\lambda_{f 0}^{+},
$$

or, if we introduce indices,

$$
\lambda_{1}\left(\mathbf{u}_{l}\right)<0<\lambda_{2}\left(\mathbf{u}_{l}\right)<\lambda_{3}\left(\mathbf{u}_{l}\right)<\cdots<\lambda_{7}\left(\mathbf{u}_{r}\right) .
$$

To the right of the stationary shock we have instead

$$
\lambda_{f}^{-}<\lambda_{A}^{-}<\lambda_{s}^{-}<0<\lambda_{0}\left(\mathbf{u}_{r}\right)<\lambda_{s}^{+}<\lambda_{A}^{+}<\lambda_{f}^{+},
$$

which, using the notation above, is equivalent to

$$
\lambda_{1}\left(\mathbf{u}_{r}\right)<\lambda_{2}\left(\mathbf{u}_{r}\right)<\lambda_{3}\left(\mathbf{u}_{r}\right)<0<\lambda_{4}\left(\mathbf{u}_{r}\right)<\cdots<\lambda_{7}\left(\mathbf{u}_{r}\right) .
$$

Clearly, for $\mathbf{u}_{l}$ and $\mathbf{u}_{r}$ above, we have

$$
\lambda_{s}^{-}<0<\lambda_{s 0}^{-} \quad \lambda_{A}^{-}<0<\lambda_{A 0}^{-},
$$

which we knew already from Figure 24a. Consider the auxiliary inequality for $k=3$. Since we have

$$
\lambda_{2}\left(\mathbf{u}_{l}\right)=\lambda_{\boldsymbol{A} 0}^{-}>0 \quad \lambda_{4}\left(\mathbf{u}_{r}\right)=\lambda_{0}\left(\mathbf{u}_{r}\right)>0,
$$
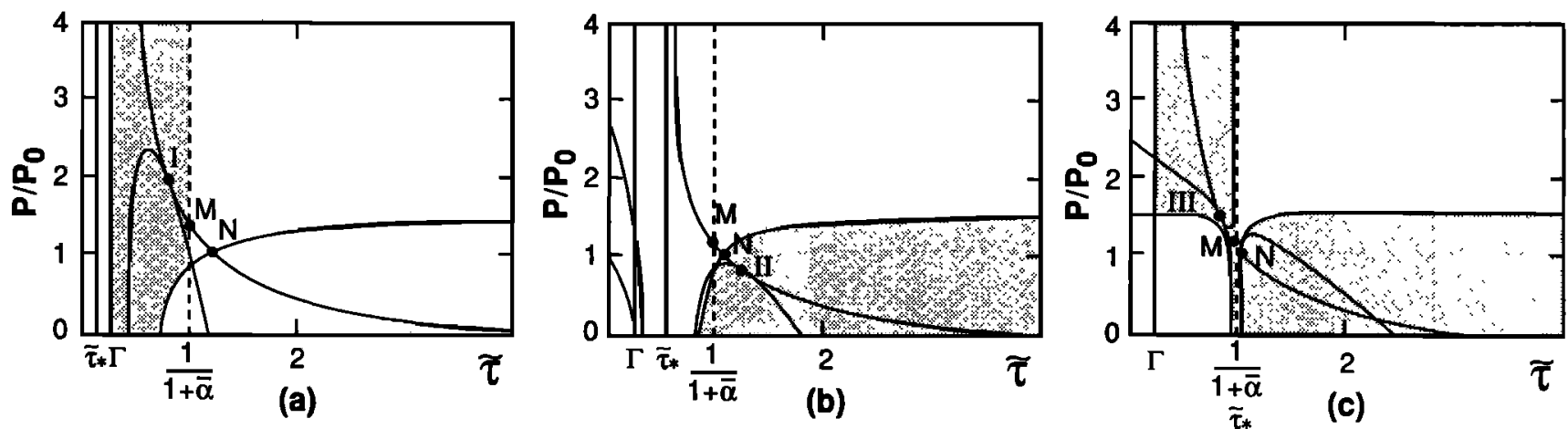

Fig. 23. The Hugoniot and Rayleigh curves appropriate to Figure 22. 


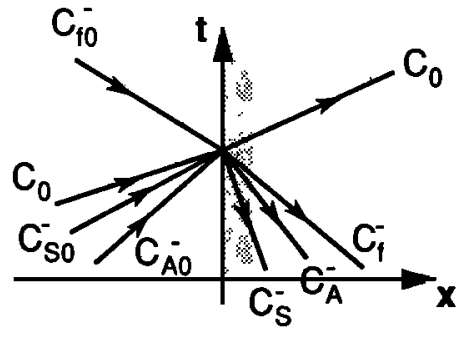

(a)

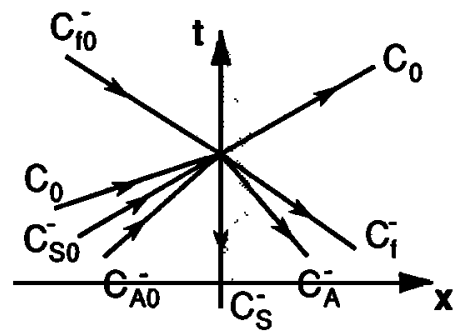

(b)

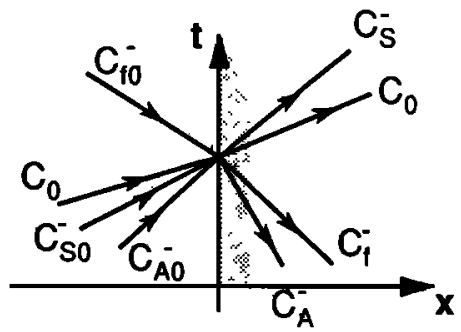

(c)

Fig. 24. Space-time diagrams for the $\theta_{0}=45^{\circ}$ example. Note the existence of two families of characteristics $\left(C_{A}^{-}\right.$ and $C_{s}^{-}$) separated by the stationary left-facing mass-loading front, both of which can be traced back to the initial data. See text for details.

the second of Lax's inequalities (66) cannot hold, and so 3-shocks (i.e., slow mode shocks) are inadmissible. Similarly, for $k=2$, we have

$$
\lambda_{1}\left(\mathbf{u}_{l}\right)=\lambda_{f 0}^{-}<0 \quad \lambda_{3}\left(\mathbf{u}_{r}\right)=\lambda_{s}^{-}<0,
$$

thereby illustrating that 2-shocks are also inadmissible. A similar conclusion is easily drawn for $u_{x}=V_{s}$ (Figure $24 b$ ). Thus the section of the shock polar curve described by (67) and (68) does not admit stable solutions; instead such downstream states are likely to disintegrate spontaneously into intermediate and slow mode mass-loading shocks. Indeed, an argument similar to that made above is seen to hold for the upper intermediate section of the classical MHD shock polar curve of section 2 .

One can go through a similar analysis, but now for the states described by Figure $24 c$, and easily convince oneself that the inequalities (66) and the geometric entropy definition at the beginning of section 4 are identical. This is left to the interested reader. It is also apparent that the region $V_{s 0}<$ $u_{x 0}<V_{A 0}<V_{f 0}, u_{x}<V_{s}$ describes admissible solutions to the mass-loading $\mathrm{R}-\mathrm{H}$ conditions and that these solutions correspond to classical slow mode shocks. Rather than examine each region of the shock polar curve in detail, we summarize the results in Figure 25.

\subsection{Halley Parameters}

In concluding this section we present some results based on parameters obtained on the inbound and outbound bow shock crossings by the Giotto mission. These results should be regarded with some caution since it is difficult to infer precise values of, for example, the shock obliquity, the mass-loading rate, and the transverse flow velocity, from the in situ measurements [e.g., Coates et al., 1990a; Neubauer et al., 1990]. Nevertheless, the Halley shock observed on the inbound crossing is thought to be quasi-perpendicular, with a shock thickness of $\sim 45,000 \mathrm{~km}$ and an upstream plasma beta of at least 5 . We have therefore chosen the following parameters for the inbound shock: $\theta_{0}=75^{\circ}, \gamma=5 / 3, \bar{\alpha}=$ $0.001, \bar{V}_{y}=0.1$, and $\beta_{p 0}=5$. The shock polar curves and the other downstream quantities are illustrated in Figure 26. Owing to the very small tangential flow velocity $\vec{V}_{y}$ and mass-loading term $\bar{\alpha}$, the properties of slow mode and intermediate mass-loading shocks are quite similar to their classical counterparts. Nevertheless, the topology of the mass-loading shock polar curve is identical to that of the $\theta_{0}=45^{\circ}$ case above, whose slow mode and intermediate shock properties are summarized in Figure 25. The inbound bow shock parameters above give $\left(M_{-}^{0}\right)^{2}=0.82$ and $\left(M_{+}^{0}\right)^{2}$ $=76.3$. Coates et al. [1990a] have suggested that the observed inbound Halley shock possessed an upstream fast Mach number $M_{f 0}=u_{x 0} / V_{f 0}$ in the range $M_{f 0} \sim 1.1-1.8$. In Figure 26 this translates as $M_{A 0}^{2} \sim 92.3-247.2$, and it is clear that two different types of stable mass-loading shocks are possible, the first being the mass-loading analogue of the fast mode MHD shock, and the second the fast mode compound shock. Given the observed downstream velocity dips and recoveries, it is conceivable that what was observed at comet Halley was in fact a train of fast mode compound shocks.

The difficulty in testing this hypothesis comes back to determining the correct upstream normal to the shock. A variety of approaches have been developed [Réme et al., 1987; Fuselier et al., 1987; Neubauer et al., 1986; Viñas and Scudder, 1986; Coates et al., 1990a; Huddleston et al., 1990]. With the exception of the last three sets of authors, the determination of the shock normal has been based on a simple paraboloid model. Huddleston et al. [1990] used instead the solutions of BBS and Galeev et al. [1985] in an effort to match the mass-loading rate to the point at which the flow was predicted theoretically to undergo a selfreversal. On the other hand, Coates et al. [1990a] utilized

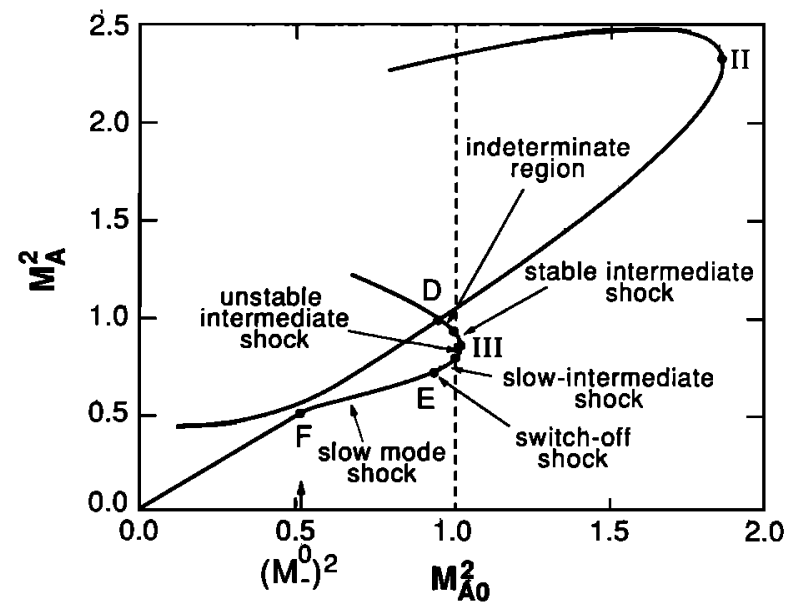

Fig. 25. Detail of the "slow" section of the $\theta_{0}=45^{\circ}$ shock polar curve. The various possible classes of shocks corresponding to each section of the shock polar curve are marked. Note in particular the sections corresponding to the slow-intermediate shock and to the stable and unstable intermediate shocks. 

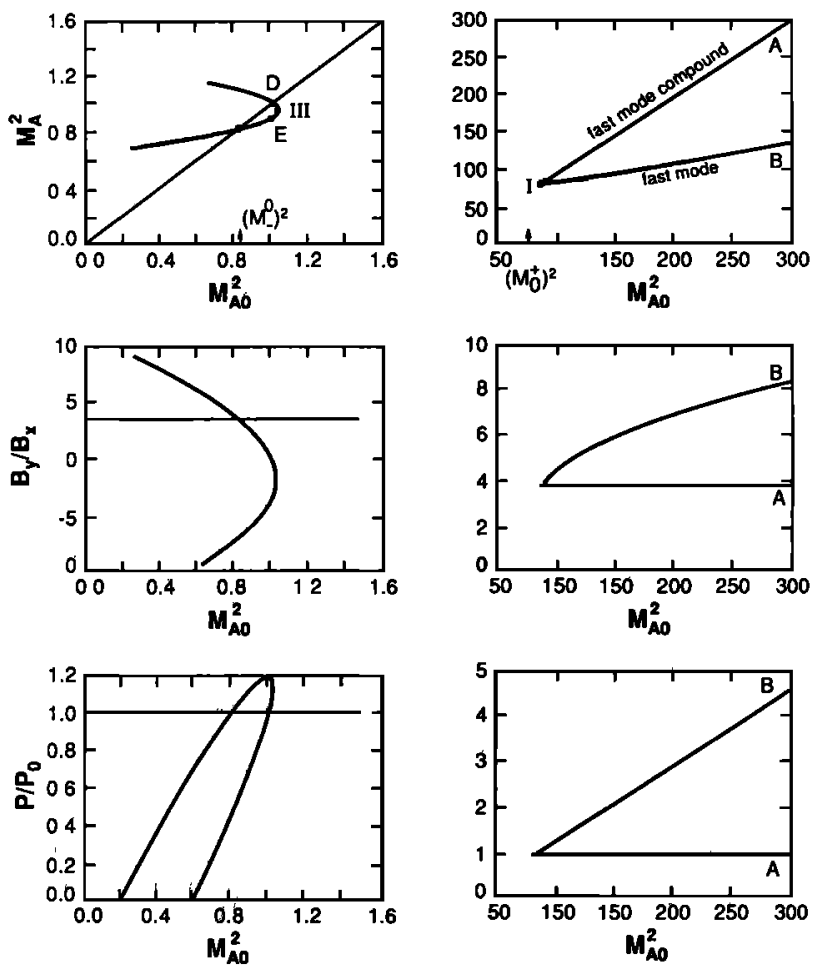

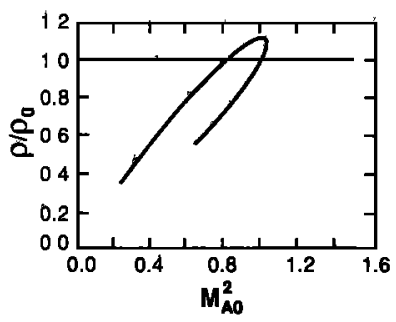

(a)

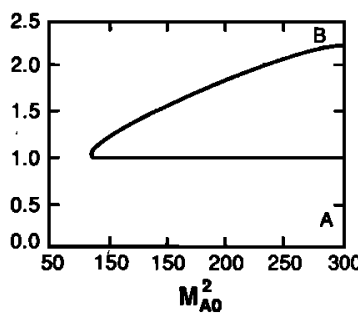

(b)
Fig. 26. The shock polar curves for $(a)$ the slow and $(b)$ the fast sections, together with the corresponding downstream MHD quantities, for parameters appropriate to the Halley bow shock observed on the inbound Giotto encounter.

the method of Viñas and Scudder [1986], which is based on the Rankine-Hugoniot relations directly, but without the addition of mass within the shock. On the basis of the analysis presented in this paper, this may in fact be a drawback to the method, particularly if the observed shock is thought to fall in what we have called the weak compression range. That this appears to be case, at both the inbound and the outbound Halley shock crossing, does in fact suggest that a more accurate approach should include the effects of mass loading. A possibility that we have not yet mentioned, but one that was used by Neubauer et al. [1990], is to utilize the coplanarity of the magnetic field to infer the direction of the shock normal. As was demonstrated in section 3, coplanarity of the magnetic field is likely to be a fairly accurate assumption for mass-loading flows, and this may provide a reasonably simple and accurate method of determining the shock normal.

On turning now to the outbound Halley shock encounter, we can adopt the following parameter values: $\theta_{0}=12^{\circ}, \gamma=$ $5 / 3, \bar{\alpha}=0.01, \widetilde{V}_{y}=0.3$, and $\beta_{p 0}=5$, because of the greater shock thickness $(\sim 120,000 \mathrm{~km})$ [Neubauer et al., 1990]. This yields normalized slow and fast Mach numbers of $\left(M_{-}^{0}\right)^{2}=$
0.08 and $\left(M_{+}^{0}\right)^{2}=5.3$. The downstream MHD variables and the shock polar relation are plotted in Figure 27. Again, the topology is not very different from that illustrated in Figure 26, so we offer no comments except to note that Coates et al. [1990a] suggested that the incident fast Mach number of the flow lay in the range $M_{f} \sim 1.6-1.7$. This translates to $M_{A 0}^{2} \sim 12.8-15.3$, again indicating that two types of fast mode mass-loading shocks are possible.

\section{Conclusions}

A completely new class of shocks, distinct from those of classical nonreacting fluid dynamics (i.e., hydrodynamics and MHD) and of combustion theory, has been described and investigated in this and earlier papers [Zank and Oughton, 1991; Zank et al., 1991]. This class of shocks is characterized by the addition of mass within the shock transition and these mass-loading fronts are to be found at comets and, depending on the circumstances, at nonmagnetized and weakly magnetized planets such as Venus and Mars. Other possibilities include the interaction of satellites such as Titan or Io with the solar wind, and the interaction of the solar wind with the interstellar medium, although none of these ideas were pursued in this paper. Mass-loading shocks are therefore likely to be fairly common in the heliosphere and thus of importance and interest.

As in paper 1, a possible analogy between mass-loading shocks and detonation/deflagration shocks can be drawn in that a physical quantity is not conserved but rather created in crossing from the upstream to the downstream region. Thus mass-loading shocks possess some characteristics of the deflagration/detonation shocks that occur in combustion theory, and many important features (such as the existence of Chapman-Jouguet points) carry across from one theory to the other. As discussed in section 3.2, even though mass loading is a small term, its associated momentum contribution in the shock frame introduces effects of physical significance. Besides the addition of mass rather than energy, what truly distinguishes mass-loading shocks from combustion shocks is that the addition of mass within the shock induces the flow to shear. We have shown that shearing has important consequences both for the global stability of the shock and for the downstream rotation of the magnetic field. Even for a parallel shock, the sheared flow tends to drag the magnetic field on passage through the mass-loading shock, thereby imbuing the mass-loading front with the characteristics of a switch-on shock, regardless of the magnitude of the plasma beta. This result goes some way toward explaining the downstream magnetic field observations at comet Halley described by Neubauer et al. [1990], but a complete understanding will come only with a detailed analysis of the full shock structure problem. It was also shown incidentally in section 3 that provided the addition of transverse momentum by the newly born ions is sufficiently small, the coplanarity theorem should continue to hold at mass-loading shocks. That this is indeed true observationally was one of the important results reported by Neubauer et al. [1990].

In considering mass-loading shocks we have utilized methods culled from the modern theory of hyperbolic differential equations to decide on the physical relevance of different solutions to the mass-loading R-H conditions. While one can obviously discard certain classes of $\mathrm{R}-\mathrm{H}$ solutions immediately, such as those for which $m^{2}<0$, the thermodynamic 


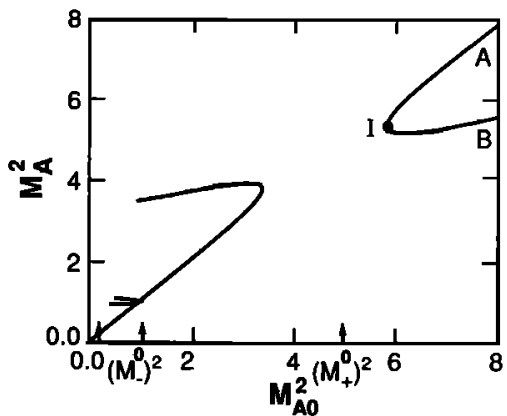

(a)

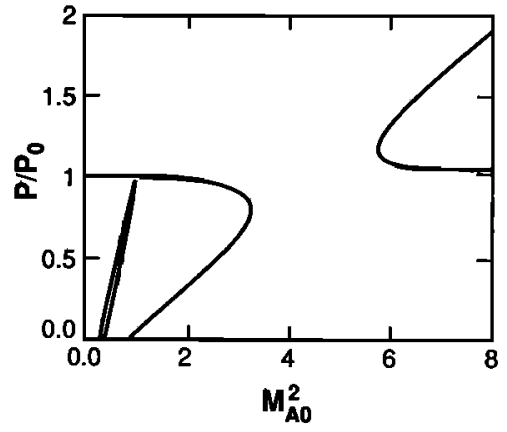

(c)

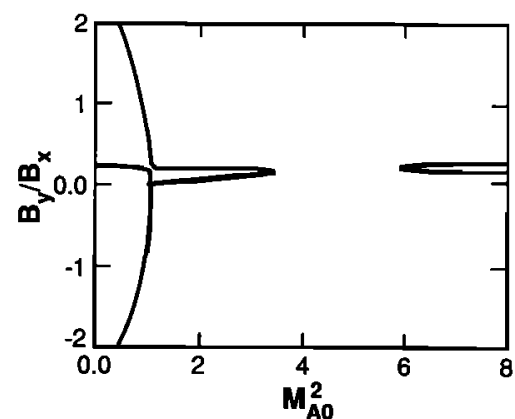

(b)

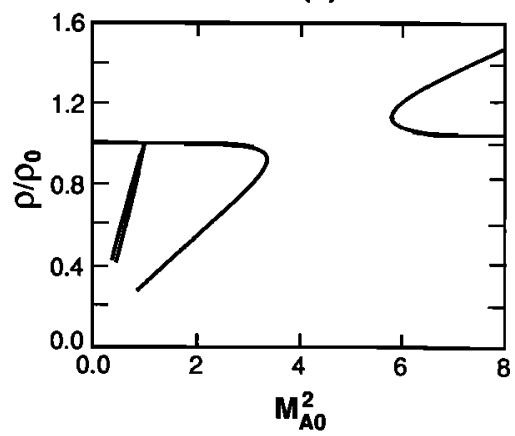

(d)

Fig. 27. The same as Figure 26, except now for the Halley shock observed on the outbound Gíotto encounter.

complexity of the mass-loading system renders classical thermodynamic methods [e.g., Landau and Lifshitz, 1979; Cabannes, 1970] ineffective, and it is not at all obvious which of the R-H solutions to retain. As has been demonstrated implicitly in this paper, the use of a geometric entropy condition (based on a set of inequalities derived by Lax [1973]) fulfills the same role for nonreacting MHD as classical entropy arguments in picking out the correct physically sensible and stable shock solutions.

As we have discussed in detail, stable mass-loading shocks analogous to classical fast mode MHD shocks exist for all parameter regimes. These fast mode mass-loading shocks have properties very similar to their classical counterparts except that the downstream gas pressure is often smaller for mass-loading shocks, particularly for parallel shocks. The reason, quite simply, is a consequence of mass loading always "twisting" the downstream magnetic field. Thus the "magnetic pressure" contribution downstream of mass-loading shocks is typically greater than is found downstream of a nonreacting fast mode shock, and so the thermal gas pressure downstream of a mass-loading front need do less work to slow the incident flow. A further consequence of mass loading is the absence of a parameter regime admitting mass-loading slow mode shocks for quasi-parallel configurations. In fact, a characteristic of the mass-loading $\mathrm{R}-\mathrm{H}$ conditions is the very large parameter regime admitting no real solutions at all. As is described below briefly, this has important implications for solving the Riemann problem in a mass-loading environment. With an increase in the obliquity of the mass-loading shock (often to nearly $45^{\circ}$ ), mass-loading analogues of the slow mode, switch-off, and intermediate shocks appear in the appropriate parameter regime of the shock polar curves. Again, these mass-loading fronts, while often fairly similar in many respects to their classical nonreacting counterparts, exhibit properties which can represent a substantial departure from their MHD analogues. For example, the switch-off shock now occurs for incident Alfvénic Mach numbers satisfying $M_{A 0}<1$. Furthermore, there can also exist a class of shocks (which we call "slowintermediate" shocks) for which $M_{A 0}<1$ and yet the downstream magnetic field is rotated in a way that is consistent with an intermediate shock.

Perhaps the most interesting of the new classes of shocks are the mass-loading fast and slow compound shocks, neither of which has a classical nonreacting MHD analogue. These structures are composed of a shock in which the incoming fluid flow is decelerated to either the fast or the slow magnetosonic speed, followed by an isentropic rarefaction front. The transition consists of a deceleration followed by an acceleration to the final downstream state, the final decelerated flow velocity being either super(fast)magnetosonic or super(slow)magnetosonic. Examination of the Giotto-Halley data at both inbound and outbound shock encounters reveals that the incident upstream state falls into the fast compound as well as the fast mode shock regime. In addition, the structure at the inbound encounter appeared to consist of a series of velocity dips and recoveries, which could be consistent with the idea of a train of fast compound shocks.

Unfortunately, as we have discussed, it is difficult to test for the existence of either fast or slow mode shocks given the complexity involved in determining accurately the shock normal from in situ observations. We have noted the possible drawbacks to the scheme of Viñas and Scudder [1986] (they exclude the possibility of mass loading within the shock), and we have suggested that the coplanar approach advocated by Neubauer et al. [1990] may be a simpler, more accurate approach. Finally, we suggest that the mass-loading $\mathrm{R}-\mathrm{H}$ relations should perhaps be used when investigating the cometary shock data.

In conclusion, we draw attention to the possibility that our 
results should be of considerable interest to those engaged in the numerical modeling of the interaction of the solar wind with comets or weakly magnetized planets (see, for example, the reviews by Spreiter and Stahara [1985] and Russell [1985]). Many sophisticated codes, such as those using piecewise parabolic [Woodward and Colella, 1984] or random choice methods [Chorin, 1977; Colella, 1982], are based upon Riemann solvers. However, the Riemann problem for massloading shocks is considerably different from that of nonreacting MHD in that the right and left waves may now be compound waves as well as shocks, slip waves, and rarefactions, and this possibility must now be incorporated into the solution of the Riemann problem. Furthermore, given the large parameter regime not admitting solutions to the $\mathrm{R}-\mathrm{H}$ conditions, it is likely that compound structures (consisting of a mixture of shocks, rarefactions, etc.) are necessary for the construction of a mass-loading transition from an arbitrary upstream to a downstream state. This may, in fact, offer yet another explanation for some of the complex structures observed at comets Halley and G-Z in regions other than at the bow shock.

\section{APPENDIX: The LAX INEQUalities}

A brief derivation of the Lax inequalities is given for a stationary discontinuity, i.e., for a discontinuity with propagation speed $s=0$. Suppose that we are given the initial conditions $\mathbf{u}(x, 0)=\mathbf{u}_{r}$ in $x>0$ and $\mathbf{u}(x, 0)=\mathbf{u}_{l}$ in $x<0$ and that the stationary discontinuity is located at $x=0$. Can these initial conditions determine the solutions $u^{i}(x, t)$ of a hyperbolic system in the half plane $t>0$ ?

Consider a hyperbolic system of $n$ equations, and let $\lambda_{1}(\mathbf{u})$ $<\cdots<\lambda_{n}(\mathbf{u})$ denote the characteristic speeds of the system (e.g., for one-dimensional gasdynamics the three eigenvalues are, of course, $\left.u \pm c_{s}, u\right)$. Now suppose that

$$
\lambda_{1}\left(\mathbf{u}_{r}\right)<\cdots<\lambda_{k}\left(\mathbf{u}_{r}\right)<0<\lambda_{k+1}\left(\mathbf{u}_{r}\right)<\cdots<\lambda_{n}\left(\mathbf{u}_{r}\right)
$$

for some index $k$. For the region $x>0, t>0$, if $i \leq k$, then since $\lambda_{i}\left(\mathbf{u}_{r}\right)<0$, the $u^{i}(0, t)$ are determined by the initial data (Figure 28), and no boundary conditions need therefore be given. Conversely, if $i>k$, then $\lambda_{i}\left(\mathbf{u}_{r}\right)>0$, and we therefore need to specify boundary conditions $u^{i}(0, t)$ for all $i=k+1, \cdots, n$ in order to determine a solution in the full quarter plane $x>0, t>0$. Thus we must specify $(n-k)$ conditions on the right boundary of the stationary discontinuity. Similarly, for the quarter plane $x<0, t>0$, it is necessary to specify $j$ conditions on the left boundary of the discontinuity if $\lambda_{j}\left(\mathbf{u}_{l}\right)<0<\lambda_{j+1}\left(\mathbf{u}_{l}\right)$. Now we have $n$ algebraic equations, the Rankine-Hugoniot conditions,

$$
[f(\mathbf{u})]=0,
$$

which connect the values on both sides of the discontinuity. Since $\mathbf{u}_{l} \neq \mathbf{u}_{r}$, this reduces to $(n-1)$ conditions between $\mathbf{u}_{l}$ and $\mathbf{u}_{r}$. Thus it is necessary that

$$
(n-k)+j=n-1 \quad \text { or } \quad j=k-1 .
$$

Accordingly, we should admit a stationary discontinuity ( $\mathbf{u}_{l}$, $\mathbf{u}_{r}$ ) provided that for some index $k, 1 \leq k \leq n$, the following inequalities hold:

$$
\begin{aligned}
& \lambda_{k}\left(\mathbf{u}_{r}\right)<0<\lambda_{k+1}\left(\mathbf{u}_{r}\right) ; \\
& \lambda_{k-1}\left(\mathbf{u}_{l}\right)<0<\lambda_{k}\left(\mathbf{u}_{l}\right) .
\end{aligned}
$$

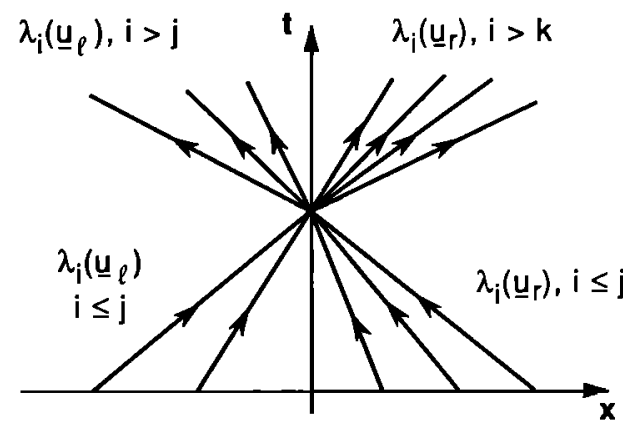

Fig. 28. The graphical determination of the boundary conditions appropriate to a stationary shock. See text for details.

Inequalities (75) are the Lax inequalities, and they can under most circumstances be given a simple geometric interpretation, as is done in the body of this paper. Any front or discontinuity satisfying (75) is called a $k$ shock, and Lax showed that use of (75) in the case of gasdynamics was formally equivalent to using standard thermodynamic arguments applied to a shock transition [e.g., Landau and Lifshitz, 1979]. Thus the inequalities (75) define an entropy condition, which can, of course, be expressed in the equivalent form

$$
\begin{gathered}
\lambda_{k}\left(\mathbf{u}_{r}\right)<0<\lambda_{k}\left(\mathbf{u}_{l}\right) ; \\
\lambda_{k-1}\left(\mathbf{u}_{l}\right)<0<\lambda_{k+1}\left(\mathbf{u}_{r}\right) .
\end{gathered}
$$

Moreover, as discussed by, for example, Liberman and Velikovich [1986], the inequalities (75) define the conditions for the existence of stable shocks.

Acknowledgments. The work of G.P.Z. and S.O. has been supported in part by the Bartol NASA Space Physics Theory Program NAGW-2076 and that of G.M.W. by NASA grant NSG7101 and NSF grant ATM-8317701. F.M.N. thanks N.F. Ness for his hospitality while visiting the BRI. We thank N. F. Ness and J. Scudder for useful discussions.

The Editor thanks A. J. Coates and another referee for their assistance in evaluating this paper.

\section{REFERENCES}

Akhiezer, A. I., G. I. Liubarskii, and R. V. Polovin, The stability of shock waves in magnetohydrodynamics, Sov. Phys. JETP, Engl. Transl., 8, 1024, 1959.

Anderson, J. E., Magnetohydrodynamic Shock Waves, MIT Press, Cambridge, Mass., 1963.

Axford, W. I., The interaction of solar wind with comets, Planet. Space Sci., 12, 719, 1964.

Bame, S. J., et al., Comet Giacobini-Zinner: Plasma description, Science, 232, 356, 1986.

Biermann, L., Kometenschweife und Solare Korpuskularstrahlung, Z. Astrophys., 29, 274, 1951.

Biermann, L., B. Brosowski, and H. U. Schmidt, The interaction of the solar wind with a comet, Sol. Phys., 1, 254, 1967.

Breus, T. K., The solar wind interaction with Mars over the solar cycle: A post-Phobos view, in Venus and Mars: Atmospheres, Ionospheres, and Solar Wind Interactions, Geophys. Monogr. Ser., vol. 66, edited by J. G. Luhmann, M. Tatrallyay, and R. O. Pepin, p. 387, AGU, Washington, D. C., 1991.

Breus, T. K., and A. M. Krymskii, Turbulent pick-up of new born ions at Venus and Mars and problems of numerical modeling of the solar wind interaction with these planets, I, Features of the solar wind interaction with planets, Planet. Space Sci., 40, 121, 1992.

Cabannes, H., Theoretical Magnetofluiddynamics, Academic, San Diego, Calif., 1970. 
Chorin, A. J., Random choice methods with applications to reacting gas flow, J. Comput. Phys., 25, 257, 1977.

Coates, A., et al., Giotto measurements of cometary and solar wind plasma at the comet Halley bow shock, Nature, 327, 489, 1987a.

Coates, A., et al., Solar wind flow through the comet Halley bow shock, Astron. Astrophys., 187, 55, $1987 b$.

Coates, A., A. D. Johnstone, R. L. Kessel, D. E. Huddleston, B. Wilken, K. Jockers, and F. M. Neubauer, Plasma parameters near the comet Halley bow shock, J. Geophys. Res., 95, 20,701, 1990a.

Coates, A., B. Wilken, A. D. Johnstone, K. Jockers, K.-H. Glassmeier, and D. E. Huddleston, Bulk properties and velocity distributions of water group ions at comet Halley: Giotto measurements, J. Geophys. Res., 95, 10,249, $1990 b$.

Colella, P., Glimm's method for gas dynamics, SIAM J. Sci. Stat. Comput., 3, 76, 1982.

Cravens, T. E., Plasma processes in the inner coma, in Comets in the post-Halley Era, edited by R. L. Newburn, M. Neugebauer, and J. Rahe, p. 1211, Kluwer Academic, Boston, Mass., 1991.

Freistühler, H., Some remarks on the structure of intermediate magnetohydrodynamic shocks, J. Geophys. Res., 96, 3825, 1991.

Fuselier, S. A., K. A. Anderson, H. Balsiger, K.-H. Glassmeier, B. E. Goldstein, M. Neugebauer, H. Rosenbauer, and E. G. Shelley, The foreshock region upstream from the comet Halley bow shock, Eur. Space Agency Spec. Publ., SP-278, 77, 1987.

Galeev, A. A., Plasma processes in the outer coma, in Comets in the post-Halley Era, edited by R. L. Newburn, M. Neugebauer, and J. Rahe, p. 1145, Kluwer Academic, Boston, Mass., 1991.

Galeev, A. A., and I. Kh. Khabibrakhmanov, Gradient catastrophe of the supersonic mass-loaded magnetized plasma flow and the formation of bow shock, Eur. Space Agency Spec. Publ., SP-311, 95, 1990.

Galeev, A. A., T. E. Cravens, and T. I. Gombosi, Solar wind stagnation near comets, Astrophys. J., 289, 807, 1985.

Hau, L.-N., and B. U. Ö. Sonnerup, On the structure of resistive MHD intermediate shocks, J. Geophys. Res., 94, 6539, 1989.

Huddleston, D. E., A. D. Johnstone, and A. J. Coates, Determination of comet Halley gas emission characteristics from mass loading of the solar wind, J. Geophys. Res., 95, 21, 1990.

Ip, W.-H., and W. I. Axford, The plasma, in Physics and Chemistry of Comets, edited by W. F. Huebner, p. 177, Springer-Verlag, New York, 1989.

Kantorwitz, A. R., and H. E. Petschek, MHD characteristics and shock waves, in Plasma Physics in Theory and Application, edited by W. B. Kunkel, p. 148, McGraw-Hill, New York, 1966.

Kennel, C. F., R. D. Blandford, and C. C. Wu, Structure and evolution of small amplitude intermediate shock waves, Phys. Fluids $B, 2,253,1989$.

Khabibrakhmanov, I. Kh., A. J. Coates, and V. L. Galinsky, Singularities in mass-loaded MHD flow: The cometary bow shock, Geophys. Res. Lett., 18, 1509, 1991.

Krankowsky, D., et al., In situ gas and ion measurements at comet Halley, Nature, 321, 347, 1986.

Landau, L. D., and E. M. Lifshitz, Fluid Mechanics, Pergamon, Elmsford, N.Y., 1979.

Lax, P. D., Hyperbolic Systems of Conservation Laws and the Mathematical Theory of Shock Waves, Reg. Conf. Ser. Appl. Math., Society for Industrial and Applied Mathematics, Philadelphia, Pa., 1973.

Liberman, M. A., and A. L. Velikovich, Physics of Shock Waves in Gases and Plasmas, Springer-Verlag, New York, 1986.

Luhmann, J. G., The solar wind interaction with Venus, Space Sci. Rev., 44, 241, 1986.

Luhmann, J. G., and L. H. Brace, Near-Mars space, Rev. Geophys., 29, 121, 1991.

Neubauer, F. M., et al., First results from the Giotto magnetometer experiment at comet Halley, Nature, 321, 352, 1986.

Neubauer, F. M., K.-H. Glassmeier, M. H. Acuña, F. Mariani, N. F. Ness, and A. J. Coates, Giotto magnetic field observations at the outbound quasi-parallel bow shock of comet Halley, Ann. Geophys., 8, 463, 1990.

Neugebauer, M., et al., The variation of protons, alpha particles, and the magnetic field across the bow shock of comet Halley, Geophys. Res. Lett., 14, 995, 1987a.

Neugebauer, M., et al., The pick-up of cometary protons by the solar wind, Astron. Astrophys., 187, 21, $1987 b$.
Neugebauer, M., B. E. Goldstein, H. Balsiger, F. M. Neubauer, R. Schwenn, and E. G. Shelley, The density of cometary protons upstream of comet Halley's bow shock, J. Geophys. Res., 94, 1261, 1989.

Ogino, T., R. J. Walker, and M. Ashour-Abdalla, A threedimensional MHD model simulation of the solar wind with comet Halley, J. Geophys. Res., 93, 9568, 1988.

Oleinik, O. A., Uniqueness and stability of the generalized solution of the Cauchy problem for a quasi-linear equation, Usp. Mat. Nauk, 14, 165, 1959. (Am. Math. Soc. Transl., Ser. 2, Engl. Transl., 285, 33, 1965.)

Omidi, N., and D. Winske, A kinetic study of solar wind mass loading and cometary bow shocks, J. Geophys. Res., 92, 13,409, 1987.

Papadopoulos, K., J. D. Huba, and T. Y. Lui, Collisionless coupling in the AMPTE artificial comet, J. Geophys. Res., 92, 47, 1987.

Réme, H., et al., General features of comet p/Halley: Solar wind interaction from plasma measurements, Astron. Astrophys., 187, $33,1987$.

Russell, C. T., Planetary bow shocks, in Collisionless Shocks in the Heliosphere: Reviews of Current Research, Geophys. Monogr. Ser., vol. 35, edited by B. T. Tsurutani and R. G. Stone, p. 109, AGU, Washington, D. C., 1985.

Schmidt, H. U., and R. Wegmann, Plasma flow and magnetic fields in comets, in Comets, edited by L. L. Wilkening, p. 538, University of Arizona Press, Tucson, 1982.

Schmidt-Voigt, M., Time dependent MHD simulations for cometary plasmas, Rep. MPA 354, Max-Planck-Inst. für Phys. und Astrophys., Inst. für Astrophys., Garching, Germany, 1988.

Smith, E. J., T. T. Bruce, J. A. Slavin, D. E. Jones, G. L. Siscoe, and D. A. Mendis, International Cometary Explorer encounter with Giacobini-Zinner: Magnetic field observations, Science, 232, $382,1986$.

Spreiter, J. R., and S. S. Stahara, Magnetohydrodynamic and gas dynamic theories for planetary bow waves, in Collisionless Shocks in the Heliosphere: Reviews of Current Research, Geophys. Monogr. Ser., vol. 35, edited by B. T. Tsurutani and R. G. Stone, p. 85, AGU, Washington, D. C., 1985.

Tatrallyay, M., C. T. Russell, J. G. Luhmann, A. Barnes, and J. D. Mihalov, On the proper Mach number and ratio of specific heats for modeling the Venus bow shock, J. Geophys. Res., 89, 7381, 1984.

Viñas, A. F., and J. D. Scudder, Fast and optimal solution to the "Rankine-Hugoniot problem," J. Geophys. Res., 91, 39, 1986.

Wallis, M. K., Shock-free deceleration of the solar wind?, Nature, 233, 23, 1971.

Wallis, M. K., Weakly-shocked flows of the solar wind plasma through atmospheres of comets and planets, Planet. Space Sci., $21,1647,1973$.

Winske, D., Origin of large magnetic fluctuations in the magnetosheath of Venus, J. Geophys. Res., 91, 11,951, 1986.

Woodward, P. R., and P. Colella, Numerical simulation of fluid flow with strong shocks, J. Comput. Phys., 54, 115, 1984.

Wu, C. C., On intermediate MHD shocks, Geophys. Res. Lett., 14, $668,1987$.

Zank, G. P., Weyl's theorem for MHD, J. Plasma Phys., 46, 11, 1991.

Zank, G. P., and S. Oughton, Properties of mass-loading shocks, 1, Hydrodynamic considerations, J. Geophys. Res., 96, 9439, 1991.

Zank, G. P., S. Oughton, F. M. Neubauer, and G. M. Webb, Mass-loading and parallel magnetized shocks, Geophys. Res. Lett., 18, 1809, 1991.

F. M. Neubauer, Institut für Geophysik und Meteorologie, Universität Köln, Albertus-Magnus-Platz, 5000 Köln-41, Germany.

S. Oughton and G. P. Zank, Bartol Research Institute, University of Delaware, Newark, DE 19716.

G. M. Webb, Department of Planetary Sciences, University of Arizona, Tucson, AZ 85721.

(Received July 24, 1991;

revised February 10, 1992, accepted February 13, 1992.) 